\title{
TWISTED DIFFERENTIAL STRING AND FIVEBRANE STRUCTURES
}

\author{
HISHAM SATI, URS SCHREIBER, AND JIM STASHEFF
}

\begin{abstract}
In the background effective field theory of heterotic string theory, the Green-Schwarz anomaly cancellation mechanism plays a key role. Here we reinterpret it and its magnetic dual version in terms of, differential twisted String- and differential twisted Fivebrane-structures that generalize the notion of Spinstructures and Spin-lifting gerbes and their differential refinement to smooth Spin-connections. We show that all these structures can be encoded in terms of nonabelian cohomology, twisted nonabelian cohomology, and differential twisted nonabelian cohomology, extending the differential generalized abelian cohomology as developed by Hopkins and Singer and shown by Freed to formalize the global description of anomaly cancellation problems in higher gauge theories arising in string theory. We demonstrate that the GreenSchwarz mechanism for the $H_{3}$-field, as well as its magnetic dual version for the $H_{7}$-field define cocycles in differential twisted nonabelian cohomology that may be called, respectively, differential twisted $\operatorname{Spin}(n)$-, String $(n)$ - and Fivebrane $(n)$ - structures on target space, where the twist in each case is provided by the obstruction to lifting the classifying map of the gauge bundle through a higher connected cover of $U(n)$ or $O(n)$. We show that the twisted Bianchi identities in string theory can be captured by the (nonabelian) $L_{\infty}$-algebra valued differential form data provided by the differential refinements of these twisted cocycles.
\end{abstract}

\section{Contents}

1. Introduction

2. Twisted topological structures in String theory

2.1. Twisted $\operatorname{Spin}^{c}$ structures and physical applications

2.2. Twisted String structures and physical applications

2.3. Twisted Fivebrane structures and physical applications

3. Twisted differential structures in String theory

3.1. Differential twisted cohomology

3.2. Twisted $\mathfrak{s t r i n g}(n)$ 2-connections

3.3. Twisted fivebrane $(n)$ 6-connections

Appendix A. $L_{\infty}$-algebraic notions

A.1. $\quad L_{\infty}$-Algebras and $L_{\infty}$-Algebroids

A.2. $L_{\infty}$-algebra representations and section

References

\section{INTRODUCTION}

String theory and M-theory involve various higher gauge-fields, which are locally given by differential form fields of higher degree and which are globally modeled by higher bundles with connection (higher gerbes with connection, higher differential characters) 22] [49. Some of these entities arise in terms of lifts through various connected covers of orthogonal or unitary groups. For example, an orientation of a Riemannian manifold $M$ can be given by a lifting of the classifying map riem : $M \rightarrow B \mathrm{O}$ for the tangent or frame bundle of $M$ to a map or $: M \rightarrow B$ SO. In turn, a Spin structure on $M$ can be given by a further lifting $s p: M \rightarrow B$ Spin. The existence of a Spin structure is an anomaly cancellation condition for fermionic particles propagating on $M$. The spaces $B \mathrm{O}, B \mathrm{SO}$ and $B$ Spin are the first steps in the Whitehead tower of $B O$. The next step above $B$ Spin is known as BString, with String the topological group known as the String group. 
Originally Killingback 33 defined a String structure on $M$ as a lift of the transgressed map $L M \rightarrow B L$ Spin on loop space through the Kac-Moody central extension $B \hat{L} \operatorname{Spin}(n)$. The existence of such a lift cancels an anomaly of the heterotic superstring on $M$ in the case that the gauge bundle is trivial. Later it was realized that this is captured down on $M$ by a lift of $s p: M \rightarrow B \operatorname{Spin}(n)$ to str $: M \rightarrow B \operatorname{String}(n)$ [56. A further lift fiv : $M \rightarrow B$ Fivebrane( $n$ ) through the next step in the Whitehead tower of $B \mathrm{O}(n)$ is similarly related to anomaly cancellation for the physical fivebrane on $M$ and accordingly the corresponding space is called $B$ Fivebrane $(n)$ [7].

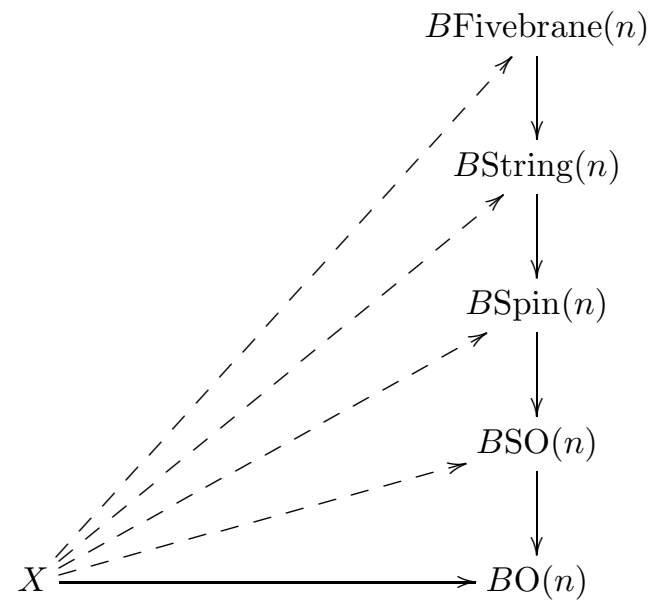

Fivebrane structure

String structure

Spin structure

Orientation

Riemannian

target space Whitehead tower of $B \mathrm{O}(n)$

FigurE 1. Topological structures generalizing $\operatorname{Spin}(n)$ structure. In application to effective background field theories appearing in string theory, these bare structures are twisted and moreover refined to differential structures.

While anomalies are canceled by these lifts of maps of topological spaces, the dynamics of these systems is controlled by smooth refinements of such maps. This is well understood for the first steps: the topological groups $O(n), S O(n)$ and $\operatorname{Spin}(n)$ naturally carry Lie group structures and the differential refinement of $X \rightarrow B \operatorname{Spin}(n)$ is well known to be given by a differential nonabelian $\operatorname{Spin}(n)$-cocycle, namely a smooth $\operatorname{Spin}(n)$-principal bundle with connection.

However, the higher connected topological groups $\operatorname{String}(n)$ and Fivebrane $(n)$ cannot be finite-dimensional Lie groups and a smooth infinite-dimensional structure for Fivebrane is not known. Moreover, even when such infinite-dimensional Lie group structures on these higher covers exist (as was recently found for the String-group [39]) by themselves they lead to the wrong smooth cohomological refinement (discussed in section 4.1 of [49]). However, $\operatorname{String}(n)$ does have a natural incarnation as a smooth 2-group [4] [27, [5] [3] 6] [48 39], a higher categorical version of a Lie group (see [37] for all general matters of higher category theory needed here and [4] for smooth higher geometry). Similarly, Fivebrane( $n)$ does naturally exist as a smooth 6-group (see section 4.1 of [49]). Generally, there are smooth $\infty$-group-refinements of all higher connected covers of Lie groups. Being smooth, these spaces have infinitesimal approximations by $L_{\infty^{-}}$ algebras in generalization of how any ordinary Lie group has a Lie algebra associated with it. Therefore, after passing to the smooth $\infty$-groupoid incarnation of the objects in the Whitehead tower of $B O$ there is a chance of obtaining differential refinements of String $(n)$ - and Fivebrane( $n$ )-structures (and beyond) that are expressed in terms of higher smooth bundles with smooth $L_{\infty}$-algebra-valued connection forms on them, and indeed these structures naturally exist [46] 21]. 
The general refinement of cohomology classes to differential cohomology classes for the case of abelian (Eilenberg-Steenrod-type) generalized cohomology theories has been discussed by Hopkins and Singer [28] and shown by Freed [22] to encode various differential (and twisted) structures in String theory. However, the cohomological structures that appear in the Freed-Witten 24] and in the Green-Schwarz anomaly cancellation mechanism [26, as well as in the magnetic dual Green-Schwarz mechanism [47] themselves originate from -and are controlled by-nonabelian structures. These are the $\mathrm{O}(n)$-principal bundle underlying the tangent bundle of spacetime and the $\mathrm{U}(n)$-principal bundle underlying the gauge bundle on spacetime, as well as their lifts to the higher connected structure groups: a map $X \rightarrow B \operatorname{Spin}(n)$ (smooth or not) gives a cocycle in nonabelian cohomology, and so do its lifts such as $X \rightarrow B \operatorname{String}(n)$ (and beyond).

Therefore, here we provide applications for the theory of (twisted) differential nonabelian cohomology, that builds on [5] [51 [52] [52, 46] and is discussed in more detail in [49. We show that the Freed-Witten and the Green-Schwarz mechanisms, as well as the magnetic dual Green-Schwarz mechanism, define differential twisted nonabelian cocycles that may be interpreted as differential twisted Spin ${ }^{c}$-, String- and Fivebranestructures, respectively. Equivalent anomalies differ by a coboundary, so that they are given by cohomologous nontrivial cocycles. Equivalence classes of anomalies are captured by the relevant cohomology. We thus have a refinement of the treatment in [4] to the twisted, smooth and differential cases.

In particular, the various abelian background fields appearing in the theory, such as the B-field and the supergravity 3-form field, are unified into a natural coherent structure with the nonabelian background fields - the spin- and gauge-connections - with which they interact. For instance, the relations between the abelian and the nonabelian differential forms that govern the Green-Schwarz mechanism [26] are realized here as a (twisted) Bianchi identity of a single nonabelian $L_{\infty}$-algebra valued connection on a smooth twisted String $(n)$-principal 2-bundle (cf. [46]). The explicit derivation of the twisted Bianchi identites of $L_{\infty}$-algebra connections corresponding to the Green-Schwarz mechanism and its magnetic dual is in section 3, with more details in section 6.2 of [21].

Summary. In this paper we achieve the following goals:

(1) generalize a Fiverbrane structure to the twisted case, and similarly for a twisted String structure;

(2) provide differential cohomology versions of these twisted structures;

(3) provide a description of the Green-Schwarz anomaly cancellation and its dual using these structures;

(4) describe the M-theory $C$-field and its dual in this context.

The first two are purely mathematical results that are of independent interest in developing higher (algebraic, geometric, topological, categorical) phenomena [21. The third and fourth are applications to (heterotic) string theory and to M-theory, respectively. We hope this will add to a better understanding of structures appearing in these theories, which in turn is hoped to result in identification of yet more rich mathematical structures within them (see 43] 44] [45] for concrete examples). From a mathematical point of view, they serve as interesting concrete examples of the formalism we have developed in the first two points.

Section 2 discusses how the anomaly cancellation mechanisms in String-theory can be understood topologically in terms of twisted higher structures given by twisted nonabelian topological cocycles. The physical examples of most relevance here arise in various anomaly cancellations in string theory. The Freed-Witten condition [24] in type IIA string theory says that the third integral Stiefel-Whitney class $W_{3}$ of a D-brane $Q$ has to be trivial relative to the Neveu-Schwarz field $\left.H_{3}\right|_{Q}$ restricted to the D-brane, in that the two classes agree: $W_{3}=\left[\left.H_{3}\right|_{Q}\right]$.

Higher versions of this example -within our point of view- are the Green-Schwarz mechanism and its magnetic dual version. Recall the notion of String structures, e.g. from [47, as maps from a space $X$ to $B \operatorname{String}(n)$, the 3 -connected cover of $B \operatorname{Spin}(n)$. In 61 the notion of twist for a String structure was considered: a space $X$ can have a twisted String structure without having a String structure, i.e. the fractional Pontrjagin class $\frac{1}{2} p_{1}(T X)$ of the tangent bundle can be nonzero while the modified class is $\frac{1}{2} p_{1}(T X)+[\beta]=0$, where $\beta: X \rightarrow K(\mathbb{Z}, 4)$ is a fixed twist for the String structure. The Green-Schwarz mechanism in string 


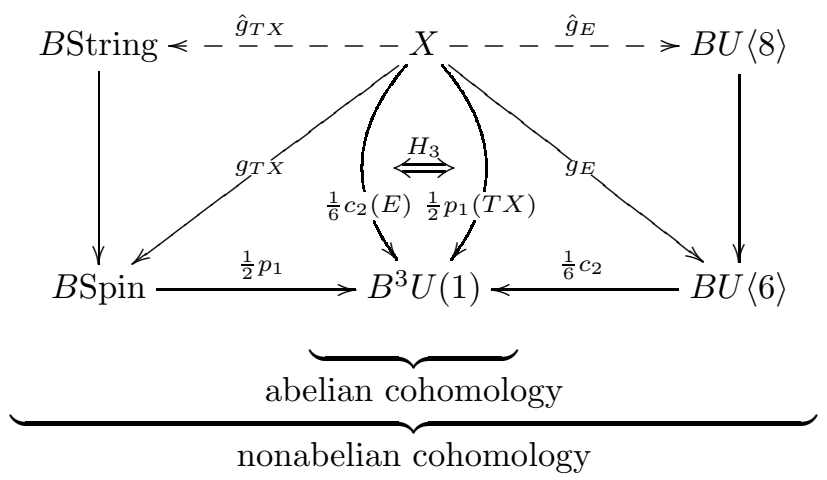

Figure 2. Abelian versus nonabelian cohomology. Since the groups String $(n)$ as well as Fivebrane $(n)$ are shifted central extension of nonabelian groups, cohomology with coefficients in these groups has abelian components but also components in nonabelian cohomology [58]. This appears as abelian cohomology twisted by nonabelian cocycles in a certain way. The Green-Schwarz mechanism implies that two classes in ordinary abelian cohomology, namely in degree four differential integral cohomology, coincide. But these classes are particularly obstruction classes to String-lifts in nonabelian cohomology. The middle part of Figure 1, labeled "abelian cohomology", identifies the cocycle representative in $H^{4}(X, \mathbb{Z})$ and the coboundary between them, but does not specify where these cocycles come from. The outer part of the diagram, labeled "nonabelian cohomology" does specify the object whose class is the one identified by the middle part. We can interpret this in ordinary homotopy theory, where it describes topological obstruction theory, but we can also interpret this after differential refinement in the $\infty$-topos [37] of smooth $\infty$-groupoids [49. In any case the morphisms in the above diagram may be interpreted as cocycles. The smooth and differential refinement we discuss in section 3 .

theory may be understood as defining a twisted String structure on target space, with twist given in terms of a classifying map for the gauge bundle.

Since a String structure is refined by a Fivebrane structure in analogy to how a String structure itself refines a Spin structure, it is natural to consider twists of Fivebrane structures in the above sense. In this paper we give a definition of twisted Fivebrane structures and show that the dual Green-Schwarz mechanism in heterotic String theory, reviewed and formalized in detail in [47, provides an example. Hence, variations on the twisted Fivebrane condition do in fact appear in string theory and in M-theory and correspond, as we will see, to anomaly cancellation conditions for the heterotic fivebrane [18] [36] and for the M-fivebrane [64 [66] 23] [16], respectively. We discuss these two cases in section 2.2.1] and section 2.3.1] in terms of topological cocycles (maps to the appropriate classifying spaces) and describe their differential refinements in section 3.2 and 3.3 .

Some time has passed between the original inception of the discussion presented in this article and the present form. The differential structures discussed here have motivated us with collaborators to further expand the general theory of higher smooth stacks and the formulation of differential cohomology in terms of these. In section 3 we survey this context and refer to various technical results obtained meanwhile.

\section{Twisted topological Structures in String theory}

We discuss here cohomological conditions arising from anomaly cancellation in String theory, for various $\sigma$-models. In each case, we introduce a corresponding notion of topological twisted structures in terms of which we interpret the corresponding anomaly cancellation condition. This prepares the ground for the 
material in section 3, where the differential refinement of these twisted structures is considered leading to the derivation of the differential anomaly-free field configurations.

The physics of all the cases we consider involves a manifold $X$-the target space- or a submanifold $Q \hookrightarrow X$ thereof - a brane- equipped with

- two principal bundles with their canonically associated vector bundles:

- a Spin-principal bundle underlying the tangent bundle $T X$ (and we will write $T X$ also to denote that Spin-principal bundle),

- and a complex vector bundle $E \rightarrow X$ - the "gauge bundle" - associated to a $S U(n)$-principal bundle or to an $E_{8}$-principal bundle with respect to a unitary representation of $E_{8}$;

- an $n$-gerbe / circle $(n+1)$-bundle with class $H^{n+2}(X, \mathbb{Z})$ representing the higher background gauge field and denoted $\left[H_{i}\right]$ or $\left[G_{i}\right]$ or similar in the following.

All these structures are equipped with a suitable notion of connetions, locally given by some differential-form data. The connection on the Spin-bundle encodes the field of gravity, that on the gauge bundle a Yang-Mills field and that on the $n$-gerbe a higher analog of the electromagnetic field.

The $\sigma$-model quantum field theory of a super-brane propagating in such a background (for instance the superstring, or the super 5-brane) has an effective action functional on its bosonic worldvolume fields that takes values, in general, in the fibers of the Pfaffian line bundle of a worldvolume Dirac operator, tensored with a line bundle that encodes the electric and magnetic charges of the higher gauge field. Only if this tensor product anomaly line bundle is trivializable is the effective bosonic action a well-defined starting point for quantization of the $\sigma$-model. Therefore, the Chern class of this line bundle over the bosonic configuration space is called the global anomaly of the system. Conditions on the background gauge fields that ensure that this class vanishes are called global anomaly cancellation conditions. These turn out to be conditions on cohomology classes that are characteristic of the above background fields. This is what we discuss in this section. Moreover, the anomaly line bundle is canonicaly equipped with a connection, induced from the connections of the background gauge fields, hence induced from their differential cohomology data. The curvature 2-form of this connection over the bosonic configuration space is called the local anomaly of the $\sigma$-model. Conditions on the differential data of the background gauge field that canonically induce a trivialization of this 2-fom are called local anomaly cancellation conditions. We consider these below in section 3 ,

The phenomenon of anomaly line bundles of $\sigma$-models induced from background field differential cohomology is classical in the physics literature, albeit in broad terms. A clear exposition is in 22. Only recently has the special case of the heterotic string $\sigma$-model for trivial background gauge bundle been made fully precise in [13, using a certain model [60] for the differential string structures that we discuss in section 3 .

2.1. Twisted $\operatorname{Spin}^{c}$ structures and physical applications. As a preparation for the twisted String- and Fivebrane structures discussed in the following sections, we consider twisted $\operatorname{Spin}^{c}$-structures and their role in anomaly cancellation for the open type II string.

2.1.1. Type II superstring on D-branes. The open type II string propagating on a Spin-manifold $X$ in the presence of a) a background $B$-field with class $\left[H_{3}\right] \in H^{3}(X, \mathbb{Z})$ and b) with endpoints fixed on a D-brane given by an oriented submanifold $Q \hookrightarrow X$, has a global worldsheet anomaly that vanishes if 24 the condition

$$
W_{3}(Q)+\left.\left[H_{3}\right]\right|_{Q}=0 \quad \in H^{3}(Q ; \mathbb{Z}),
$$

holds. Here $W_{3}(Q)$ is the third integral Stiefel-Whitney class of the tangent bundle $T Q$ of the brane and $\left[H_{3}\right]_{Q}$ denotes the restriction of $\left[H_{3}\right]$ to $Q$. Sufficiency of the condition is discussed in $[20$. 


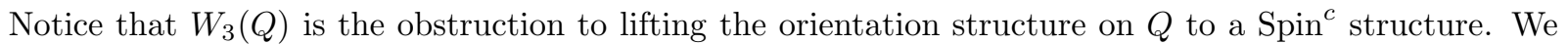
can formulate this in terms of homotopy theory as follows. There is a homotopy pullback diagram

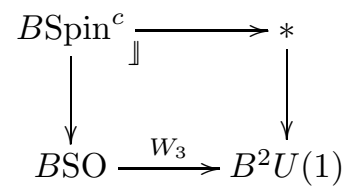

of topological spaces, where $B \mathrm{SO}$ is the classifying space of the (stable) special orthogonal group, $B^{2} U(1) \simeq$ $K(\mathbb{Z}, 3)$ is the Eilenberg-MacLane space that classifies degree-3 integral cohomology, and the continuous map denoted $W_{3}$ is a representative of the universal class $W_{3}$ under this classification.

Homotopy pullbacks and their properties play a central role in all of our discussion here, first in the category of topological spaces, and then later, in section [3, in categories of higher stacks. The reader unfamiliar with the basics of abstract homotopy theory might consult the review in section A.2 of [37. A basic fact is that a homotopy fiber of connected spaces as in (2.2), where the right vertical morphism is the point inclusion, may be computed, up to weak homotopy equivalence, as an ordinary pullback (an ordinary fiber product) after replacing the point inclusion by the path fibration. Specifically, for $X$ any pointed topological space, write $P X$ for the space of continuous paths in $X$ that end at the base point $x \in X$, and write $P X \rightarrow X$ for the projection to the other endpoint of the path. Then the ordinary pullback

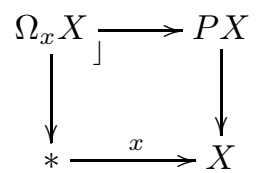

is a model for the loop space of $X$. Moreover, for $\phi: Y \rightarrow X$ any map, the ordinary pullback

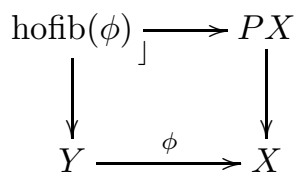

is a model for the homotopy fiber $\operatorname{hofib}(\phi)$ of $\phi$.

The homotopy pullback (2.2) exhibits the classifying space of the group $\operatorname{Spin}^{c}$ as the homotopy fiber of $W_{3}$. The universal property of the homotopy pullback says that the space of continuous maps $Q \rightarrow B$ Spin ${ }^{c}$ is the same (is weak homotopy equivalent to) the space of maps $o_{Q}: Q \rightarrow B$ SO that are equipped with a homotopy from the composite $Q \stackrel{o_{Q}}{\longrightarrow} B \mathrm{SO} \stackrel{W_{3}}{\longrightarrow} B^{3} U(1)$ to the trivial cocycle $Q \rightarrow * \rightarrow B^{3} U(1)$. In other words, for every choice of homotopy filling of the outer diagram

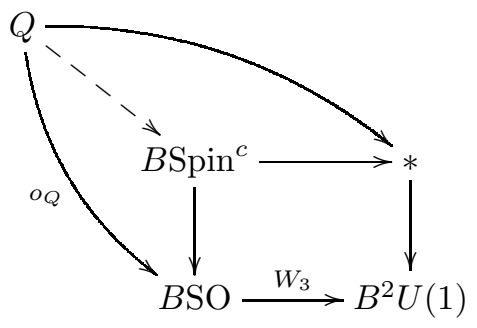

there is a contractible space of choices for the dashed arrow such that everything commutes up to homotopy. Since a choice of map $o_{Q}: Q \rightarrow B$ SO is an orienation structure on $Q$, and a choice of map $Q \rightarrow B$ Spin ${ }^{c}$ is a $\operatorname{Spin}^{c}$ structure, this implies that $W_{3}\left(o_{Q}\right)$ is the obstruction to the existence of a Spin ${ }^{c}$ structure on $Q$ (equipped with $o_{Q}$ ). Moreover, since $Q$ is a manifold (hence a $\mathrm{CW}$-complex), the functor $\operatorname{Maps}(Q,-)$ that forms mapping spaces out of $Q$ preserves homotopy pullbacks. Since $\operatorname{Maps}(Q, B S O)$ is the space of 
orientation structures, we can refine the discussion so far by noticing that the space of $\operatorname{Spin}^{c}$ structures on $Q$, denoted $\operatorname{Maps}\left(Q, B \operatorname{Spin}^{c}\right)$, is itself the homotopy pullback in the diagram

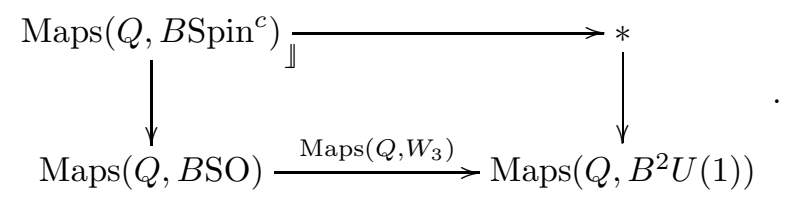

A variant of this characterization will be crucial for the definition of (spaces of) twisted such structures below.

These kinds of arguments, although elementary in homotopy theory, are of importance for the interpretation of anomaly cancellation conditions that we consider here. Variants of these arguments (first for other topological structures, then with twists, then refined to smooth and differential structures) will appear over and over again in our discussion.

In the case that the class of the $B$-field vanishes on the D-brane, $\left.\left[H_{3}\right]\right|_{Q}=0$, hence that its representative $H_{3}: Q \rightarrow K(\mathbb{Z}, 3)$ factors through the point up to homotopy, condition (2.1) states that the oriented D-brane $Q$ must admit a $\operatorname{Spin}^{c}$ structure, namely a choice of null-homotopy $\eta$ in 1$]$

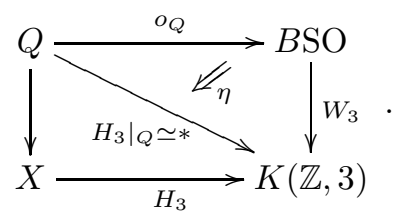

If, more generally, $\left.\left[H_{3}\right]\right|_{Q}$ does not necessarily vanish, then condition (2.1) still is equivalent to the existence of a homotopy $\eta$ in a diagram of the above form:

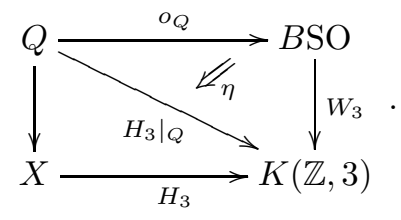

We may think of this as saying that $\eta$ still "trivializes" $W_{3}\left(o_{Q}\right)$, but not with respect to the canonical trivial cocycle, but with respect to the given reference background cocycle $\left.H_{3}\right|_{Q}$ of the $B$-field. Accordingly, following [61, we may say that such an $\eta$ exhibits not a $\operatorname{Spin}^{c}$-structure on $Q$, but an $\left[H_{3}\right]_{Q}$-twisted $\operatorname{Spin}^{c}$ structure.

For this notion to be useful, we need to say what an equivalence or homotopy between two twisted $\operatorname{Spin}^{c}$ structures is, what a homotopy between such homotopies is, etc., and hence what the space of twisted $\operatorname{Spin}^{c}$ structures is. However, by generalization of (2.6) we naturally do have such a space.

Definition 2.1. For $X$ a manifold and $[c] \in H^{3}(X, \mathbb{Z})$ a degree-3 cohomology class, we say that the space $W_{3} \operatorname{Struc}(Q)_{[c]}$ defined as the homotopy pullback

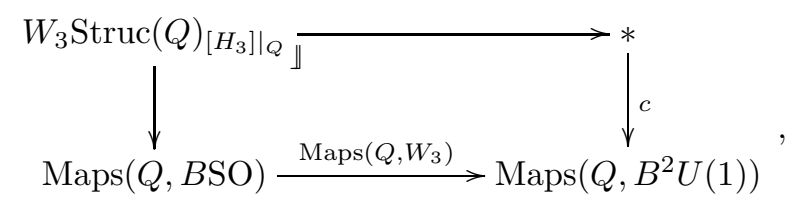

is the space of $[c]$-twisted $\operatorname{Spin}^{c}$ structures on $X$, where the right vertical morphism picks any representative $c: X \rightarrow B^{2} U(1) \simeq K(\mathbb{Z}, 3)$ of $[c]$.

\footnotetext{
${ }^{1}$ Beware that there are homotopies filling all our diagrams, but only in some cases, such as here, do we want to make them explicit and give them a name.
} 
In terms of this notion, the anomaly cancellation condition (2.1) is now read as being a requirement of existence of structure:

Observation 2.2. On an oriented manifold $Q$, condition (2.1) implies the existence of $\left.\left[H_{3}\right]\right|_{Q}$-twisted $W_{3}$ structure, provided by a lift of the orientation structure $o_{Q}$ on $T Q$ through the left vertical morphism in def. 2.1 .

This makes good sense, because that extra structure is the extra structure of the background field of the $\sigma$-model background, subjected to the condition of anomaly freedom. We will see this in more detail in the following examples, and then again in section 3 ,

2.2. Twisted String structures and physical applications. We discuss twisted String structures and their role in anomaly cancellation of

(1) the heterotic string;

(2) M-theory in the bulk;

(3) the boundary in M-theory.

2.2.1. The heterotic string. The heterotic/type I string, propagating on a Spin-manifold $X$ and coupled to a gauge field given by a Hermitean complex vector bundle $E \rightarrow X$, has a global anomaly that vanishes if the Green-Schwarz anomaly cancellation condition [26]

$$
\frac{1}{2} p_{1}(T X)-\operatorname{ch}_{2}(E)=0 \quad \in H^{4}(X ; \mathbb{Z})
$$

holds. Here $\operatorname{ch}_{2}(E)$ is the second Chern character of $E$ which reduces to the second Chern class $c_{2}(E)$ in the cases we consider, and $\frac{1}{2} p_{1}$ is given by the following classical fact (see [9]).

Fact 2.3. The first Pontrjagin class $p_{1} \in H^{4}(B S O, \mathbb{Z})$ becomes divisible precisely by 2 when pulled back to $B$ Spin. The corresponding preimage under multiplication by two, denoted $\frac{1}{2} p_{1}$, is a generator of the group $H^{4}(B$ Spin, $\mathbb{Z}) \cong \mathbb{Z}$.

As in the $\operatorname{Spin}^{c}$ case discussed above, this means that at the level of cocycles a certain homotopy exists. Here it is this homotopy which is the representative of the $B$-field to which the string couples. In detail, write $\frac{1}{2} p_{1}: B$ Spin $\rightarrow B^{3} U(1)$ for a representative of the universal first fractional Pontrjagin class, and similarly $c_{2}: B \mathrm{SU} \rightarrow B^{3} U(1)$ for a representative of the universal second Chern class, where now $B^{3} U(1) \simeq K(\mathbb{Z}, 4)$ is equivalent to the Eilenberg-MacLane space that classifies degree-4 integral cohomology. Then if $T X: X \rightarrow$ $B$ Spin is a classifying map of the Spin-bundle and $E: X \rightarrow B S U$ is one of the gauge bundles, the above anomaly cancellation condition says that there is a homotopy, denoted $H_{3}$, in the diagram

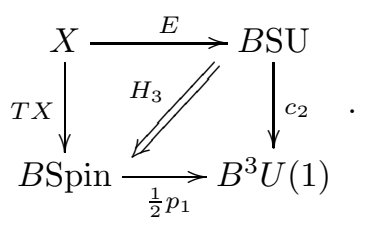

Notice that if both $\frac{1}{2} p_{1}(T X)$ as well as $c_{2}(E)$ happen to be trivial, such a homotopy is equivalently a map $H_{3}: X \rightarrow \Omega B^{3} U(1) \simeq B^{2} U(1)$. So in this special case the B-field in the background of the heterotic string is a $U$ (1)-gerbe, or a circle 2-bundle, as in the previous case of the type II string in section 2.1. Generally, the homotopy $\mathrm{H}_{3}$ in the above diagram exhibits the B-field as a twisted gerbe, whose twist is the difference class $\frac{1}{2} p_{1}(T X)-c_{2}(E)$. This is essentially the perspective adopted in 22] (in a somewhat different language).

For the general discussion of interest here it is useful to slightly shift the perspective on the twist. To that end, first consider, by analogy with (2.2), the following definition. 
Definition 2.4. The String group is the loop space of the homotopy fiber BString of the universal class $\frac{1}{2} p_{1}$ from Proposition 2.3

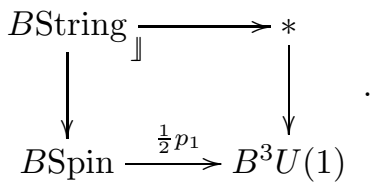

For more details on this and a collection of references see section 4.1 of [49]. Accordingly, one says that a String structure on the Spin bundle $T X: X \rightarrow B$ Spin is a homotopy filling the outer square of the diagram

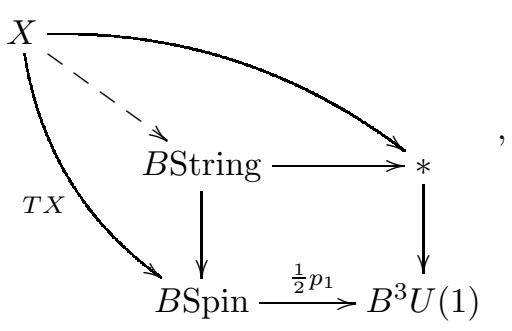

or, equivalently- by the universal property of homotopy pullbacks- a choice of dashed morphism filling the interior of this square, as indicated. 3 Therefore, now by analogy with (2.8), we say that a $c_{2}(E)$ twisted String structure is a choice of homotopy $H_{3}$ filling the diagram (2.11). This notion of twisted String structures was originally suggested in [61. For it to be useful, we need to say what homotopies of twisted String structures are, homotopies between these, etc. Hence we need to say what the space of twisted String structures is. This is what the following definition provides, analogously to Definition 2.1.

Definition 2.5. For $X$ a manifold, and for $[c] \in H^{4}(X, \mathbb{Z})$ a degree- 4 cohomology class, we say that the space of $c$-twisted String structures on $X$ is the homotopy pullback $\frac{1}{2} p_{1} \operatorname{Struc}_{[c]}(X)$ in the diagram

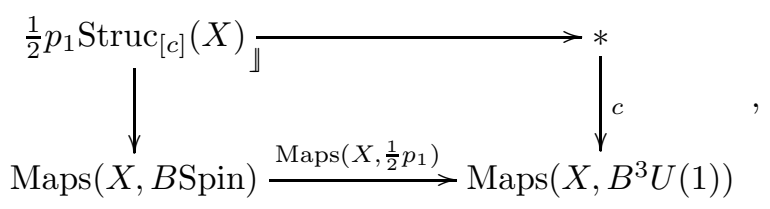

where the right vertical morphism picks a representative $c$ of $[c]$.

In terms of this then, we find

Observation 2.6. The anomaly cancellation condition (2.10) is, for a fixed gauge bundle $E$, precisely the condition that ensures a lift of the given Spin structure to a $c_{2}(E)$-twisted String structure on $X$, through the left vertical morphism of def. 2.5.

Of course the full background field content involves more than just this topological data; it also consists of local differential form data, such as a 1-form connection on the bundles $E$ and on $T X$ and a connection 2 -form on the 2-bundle whose curvature is $H_{3}$. Below, in section 3, we identify this differential anomaly-free field content with a differential twisted String structure.

\footnotetext{
${ }^{2}$ Originally, this condition was considered in its weaker incarnation after transgression to loop space 33 .
} 
The 10-dimensional string theory backgrounds discussed so far are supposed to be part of a bigger picture, which schematically looks as follows

$$
\text { UV-complete theory } \stackrel{\text { low energy approximation }}{\longrightarrow} \text { effective field theory }
$$

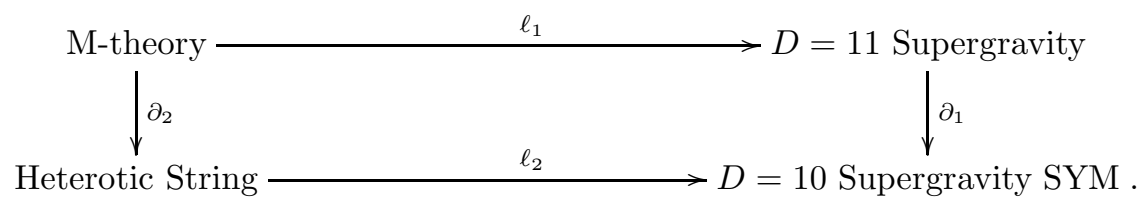

Here the bottom and right structures and maps are fairly well-defined. The right vertical map denotes the restriction of 11-dimensional supergravity on a manifold with boundary to 10-dimensional heterotic supergravity on the boundary. The bottom left morphism indicates that heterotic supergravity is the effective low energy field theory whose UV-completion is heterotic string theory. The top left entry denotes M-theory, which is what meaningfully completes this schematic diagram. Accordingly, the 2-brane and 5-brane solutions of 11-dimensional supergravity have incarnations as fundamental objects in M-theory, the M2-brane and the M5-brane.

We discuss the topological anomalies for the M2-brane, first in the bulk theory, then after restriction to the boundary, where it becomes the heterotic string of section 2.2.1. At the level of fields, this is the $C$-field in the bulk becoming the $B$-field on the boundary.

2.2.2. M-theory in the bulk. The bosonic field content of 11-dimensional supergravity on a Spin 11-manifold $Y$ consists of a Spin-bundle $T Y$ (with connection), as well as of the $C$-field, which has underlying it a 2 -gerbe - or circle 3-bundle - with class $\left[G_{4}\right] \in H^{4}(Y, \mathbb{Z})$. The M2-brane that couples to these background fields has an anomaly that vanishes if 63

$$
2\left[G_{4}\right]=\frac{1}{2} p_{1}(T Y)-2 a(E) \in H^{4}(Y, \mathbb{Z}),
$$

where $E \rightarrow Y$ is an auxiliary $E_{8}$-principal bundle, whose class $a(E)$ is defined by this condition. This is also called the quantization condition for the $C$-field.

In the absence of smooth or differential structure, since $E_{8}$ is 15-coskeletal, one could therefore replace the $E_{8}$-bundle here by a $U(1)$-2-gerbe, hence by a $B^{2} U(1)$-principal bundle, and replace condition (2.15) by

$$
2\left[G_{4}\right]=\frac{1}{2} p_{1}(T Y)-2 \mathrm{DD}_{2}
$$

where $\mathrm{DD}_{2}$ is the canonical 4-class of this 2-gerbe (the "second Dixmier-Douady class", hence the notation). While topologically this condition is equivalent, over an 11-dimensional $X$, to (2.15), the spaces of solutions of smooth refinements of these two conditions will differ, because the space of smooth gauge transformations between $E_{8}$ bundles is quite different from that of smooth gauge transformations between circle 2-bundles. In the Hořava-Witten reduction [29] of the 11-dimensional theory down to the heterotic string in 10 dimensions, this difference is supposed to be relevant, since the heterotic string in 10 dimensions sees the smooth $E_{8^{-}}$ bundle with connection.

In either case, we can understand the situation as a refinement of that described by (twisted) Stringstructures, as above, via a higher analogue of the passage from Spin-structures to Spin ${ }^{c}$-structures, as in section 2.1. To that end, notice the following fact, which provides an alternative perspective on (2.2), and which uses the point of view on twisted structures advocated recently in [44] [45.

Proposition 2.7. The classifying space BSpin ${ }^{c}$ is the homotopy fiber product of a representative of the universal second Stiefel-Whitney class $w_{2} \in H^{2}\left(B S O, \mathbb{Z}_{2}\right)$ with a representative of the mod 2-reduction of 
the universal first Chern class $c_{1} \in H^{2}(B U(1), \mathbb{Z})$

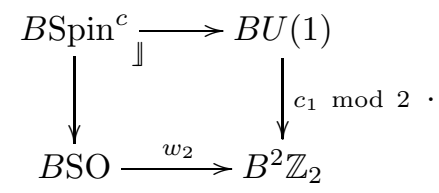

This remains true after refinement to smooth and differential structures, as we discuss below in 3 . Due to the universal property of the homotopy pullback, this says, in particular, that a lift from an orientation structure to a Spin ${ }^{c}$-structure is a cancelling by a Chern class of the class obstructing a Spin-structure. In this way, lifts from orientation structures to Spin ${ }^{c}$-structures are analogous to the divisibility condition (2.15), since in both cases the obstruction to a further lift through the Whitehead tower of the orthogonal group is absorbed by a universal "unitary" class.

In higher analogy to this situation, and in the spirit of [44] [45, we therefore have the following definition.

Definition 2.8. For $G$ some topological group, and $\alpha: B G \rightarrow K(\mathbb{Z}, 4)$ a universal 4-class, we say that String $^{\alpha}$ is the loop group of the homotopy pullback 3

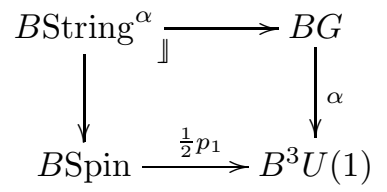

of $\alpha$ along a representative of the first fractional Pontrjagin class $\frac{1}{2} p_{1} \in H^{4}(B \operatorname{Spin}, \mathbb{Z})$.

Of relevance for the present purpose are the following cases. Notice that on the space $B^{2} U(1) \simeq K(\mathbb{Z}, 3)$, which classifies circle 2-bundles / $U(1)$-bundle gerbes, the canonical 3-class is traditionally called the DixmierDouady class, denoted DD. Accordingly, it makes sense to speak of the canonical 4 -class on $B^{3} U(1) \simeq$ $K(\mathbb{Z}, 4)$, which classifies circle 3 -bundle / $U(1)$-bundle 2-gerbes as the second Dixmier-Douady class $\mathrm{DD}_{2}$. We are interested in the case when the first Spin characteristic class $\lambda=\frac{1}{2} p_{1}$ is divisible by 2 , so that we can 'divide by 2 ' in equation (2.16). For $\alpha=\mathrm{DD}_{2}$ we have that a Spin-structure lifts to a $\mathrm{String}^{2 \mathrm{DD}_{2}}$-structure precisely if $\frac{1}{2} p_{1}$ is further divisible by 2 . Equivalently, a Spin-principal bundle $P$ lifts to a $\mathrm{String}^{2 \mathrm{DD}}{ }_{2}-$ principal bundle precisely if the corresponding Chern-Simons 2-gerbe $\frac{1}{2} p_{1}(P)$ is twice some other 2 -gerbe. Equivalently this says that, the fourth Stiefel-Whitney class vanishes, $w_{4}=0$, because, as explained in 43 . [44], $w_{4}$ is the mod 2 reduction of the integral first Spin characteristic class $\lambda=\frac{1}{2} p_{1}$. In summary, we have for a given Spin-structure $Y \rightarrow B$ Spin the following diagram

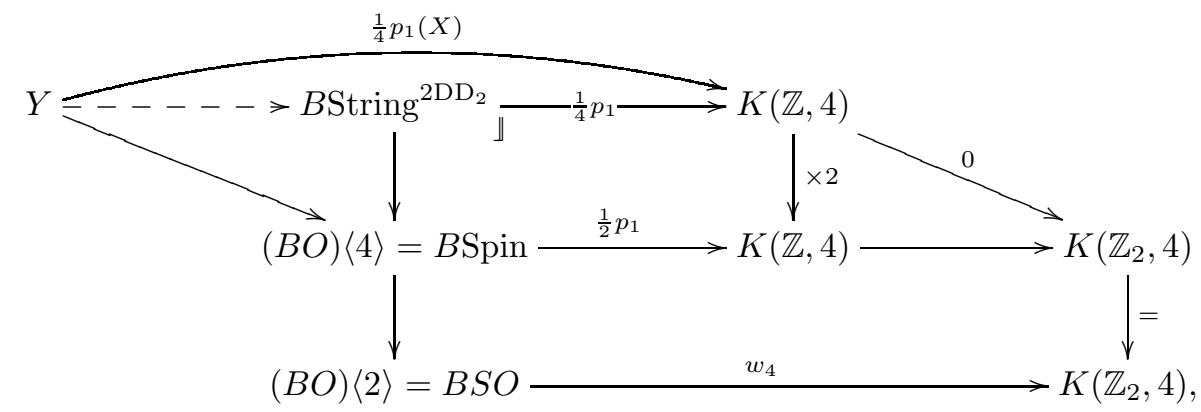

where the dashed arrow is a lift of the given Spin-structure.

Observation 2.9. The class $\frac{1}{4} p_{1}$ is the obstruction to lifting a String ${ }^{2 \mathrm{DD}_{2}}$ bundle, to a String-bundle. Here, String $^{2 \mathrm{DD}_{2}}$ is the loop space of the space $B \operatorname{String}^{2 \mathrm{DD}_{2}}$, defined above.

\footnotetext{
${ }^{3}$ We are using the notation String ${ }^{\alpha}$ to distinguish from the notion of String ${ }^{c}$ structure (related to Spin ${ }^{c}$ in the same way that String is related to Spin) studied in [45. The latter is a special case of the former, as explained in op. cit.
} 
Proof. The pasting law for homotopy pullback implies that the homotopy fiber of $\frac{1}{4} p_{1}$ is $B$ String.

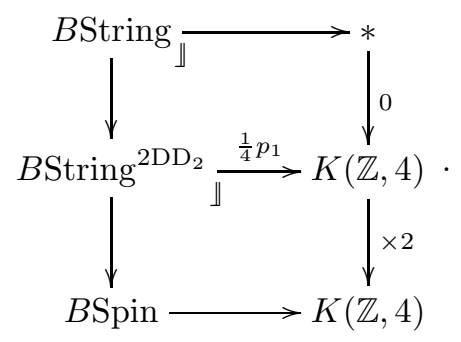

This observation is analogous to proposition 2 in [47] for the Fivebrane case, where there we were considering the comparison of $\frac{1}{48} p_{2}$ to the obstruction to Fivebrane structure, given by $\frac{1}{6} p_{2}$. We will discuss this further later in the paper.

Similarly, with $a: B E_{8} \rightarrow B^{3} U(1)$ the canonical universal 4-class for $E_{8}$-bundles and $X$ a manifold of dimension $\operatorname{dim} X \leq 14$ we have that a Spin-structure on $X$ lifts to a String $^{2 a}$-structure precisely if $\frac{1}{2} p_{1}$ is further divisible by 2 .

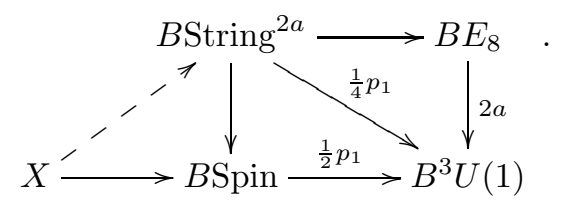

Using this we can now reformulate the anomaly cancellation condition (2.15) as follows.

Definition 2.10. For $X$ a manifold and for $[\alpha] \in H^{4}(X, \mathbb{Z})$ a cohomology class, the space $\left(\frac{1}{2} p_{1}-2 a\right) \operatorname{Struc}[\alpha](X)$ of $[\alpha]$-twisted String $^{2 a}$-structures on $X$ is the homotopy pullback

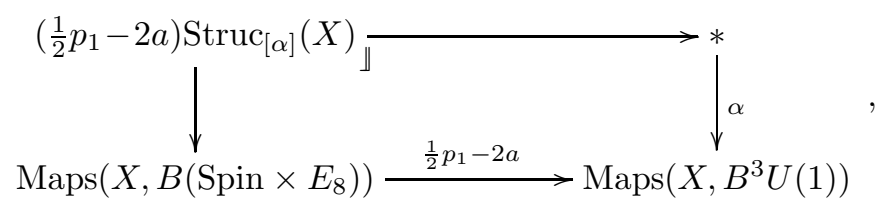

where the right vertical map picks a cocycle $\alpha$ representing the class $[\alpha]$.

This can be viewed as a "twist for the twisted String structure". In terms of this definition, we have

Observation 2.11. Condition (2.15) is precisely the conditon guaranteeing a lift of the given Spin- and the given $E_{8}$-principal bundle to a $\left[G_{4}\right]$-twisted String $^{2 a}$-structure through the left vertical map from def. 2.10 .

2.2.3. M-theory with boundary: Heterotic M-theory. In [29], Horava and Witten carefully analyzed the map $\partial_{1}$ in diagram (2.14) and gave arguments on how it must extend to $\partial_{2}$. If we denote by $Q:=\partial Y \hookrightarrow Y$ the boundary inclusion, then the condition they find is a boundary condition on the $C$-field, saying that the restriction of its 4-class to $Q$ has to vanish,

$$
\left.\left[G_{4}\right]\right|_{Q}=0
$$

This implies that over $Q$ the anomaly-cancellation condition (2.15) becomes

$$
\left.\frac{1}{2} p_{1}(T Y)\right|_{Q}=\left.2 a(E)\right|_{Q} \in H^{4}(Q, \mathbb{Z})
$$


This is equivalent, in direct analogy with (2.8), to the existence of a homotopy of the form

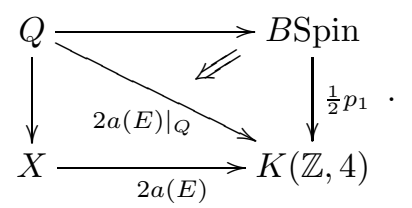

Essentially in this form twisted string structures in the context of string theory were proposed in (the first eprint version of) 61]. We see here a general pattern of twisted structures occuring as relative trivializations on branes.

Notice that on $Q$ this is the Green-Schwarz anomaly cancellation condition (2.10) of the heterotic string, but refined by a further cohomological divisibility condition. The following statement says that this may equivalently be reformulated in terms of String ${ }^{2 a}$ structures.

Proposition 2.12. For $E \rightarrow Y$ a fixed $E_{8}$-bundle, we have an equivalence

$$
\left.\operatorname{Maps}\left(Y, B \operatorname{String}^{2 a}\right)\right|_{E} \simeq\left(\frac{1}{2} p_{1}\right) \operatorname{Struc}(Y)_{[2 a(E)]}
$$

between, on the right, the space of $2 a(E)$-twisted String-structures from def. 2.5. and, on the left, the space of String $^{2 a}$-structures with fixed class $2 a$, hence the homotopy pullback $\operatorname{Maps}\left(Y, B \operatorname{String}^{2 a}\right) \times_{\operatorname{Maps}\left(Y, B E_{8}\right)}\{E\}$.

Proof. Consider the diagram

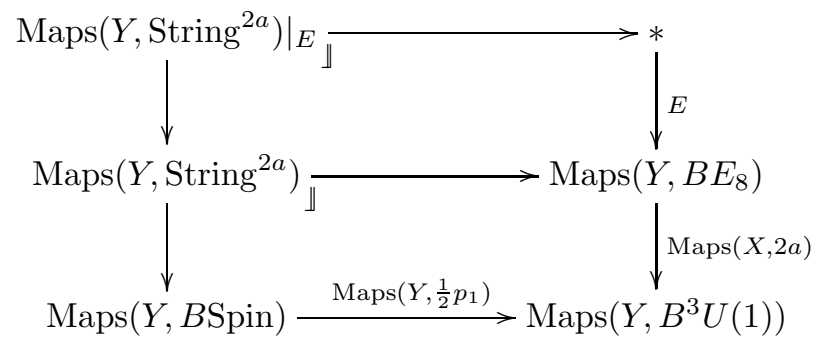

The top square is a homotopy pullback by definition. Since $\operatorname{Maps}(Y,-)$ preserves homotopy pullbacks (for $Y$ a manifold, hence a CW-complex), the bottom square is a homotopy pullback by definition 2.8. Therefore, by the pasting law, also the total rectangle is a homotopy pullback. With def. 2.5 this implies the claim.

Therefore the boundary anomaly cancellation condition for the M2-brane has the following equivalent formulation.

Observation 2.13. For $X$ a Spin-manifold equipped with a complex vector bundle $E \rightarrow Y$, condition (2.20) precisely guarantees the existence of a lift to a String ${ }^{2 a}$-structure through the left vertical map in the proof of prop. 2.12.

2.3. Twisted Fivebrane structures and physical applications. We discuss twisted fivebrane structures and their role in anomaly cancellation in

(1) the NS-5-brane and dual heterotic string theory;

(2) the M5-brane.

2.3.1. The NS-5-brane. The magnetic dual of the (heterotic) string is the NS-5-brane. Where the string is electrically charged under the $B_{2}$-field with class $\left[H_{3}\right] \in H^{3}(X, \mathbb{Z})$, the NS-5-brane is electrically charged under the $B_{6}$-field with class $\left[H_{7}\right] \in H^{7}(X, \mathbb{Z})[14$. As we discuss in detail shortly, in the presence of a String-structure, hence when $\frac{1}{2} p_{1}(T X)=0$, the anomaly of the 5 -brane $\sigma$-model vanishes if the background fields satisfy

$$
\frac{1}{6} p_{2}(T X)=8 \operatorname{ch}_{4}(E) \in H^{8}(X, \mathbb{Q})
$$


where $E$ is an $E_{8} \times E_{8^{-}}$or $\operatorname{Spin}(32) / \mathbb{Z}_{2}$-principal bundle, and $\operatorname{ch}(E)$ denotes its Chern character, and where $\frac{1}{6} p_{2}$ denotes the second fractional Pontrjagin class given by the following classical fact (see [9]).

Fact 2.14. The second Pontrjagin class $p_{2} \in H^{8}(B \mathrm{SO}, \mathbb{Z})$ becomes divisible precisely by 6 when pulled back to BString. The corresponding preimage under multiplication by six, denoted $\frac{1}{6} p_{2}$, is a generator of the group $H^{8}(B$ Spin, $\mathbb{Z}) \cong \mathbb{Z}$.

It is clear now that a discussion entirely analogous to that of section 2.2.1 applies. For the untwisted case the following terminology was introduced in [47].

Definition 2.15. Write Fivebrane for the loop group of the homotopy fiber BFivebrane of a representative $\frac{1}{6} p_{2}$ of the universal second fractional Pontrjagin class

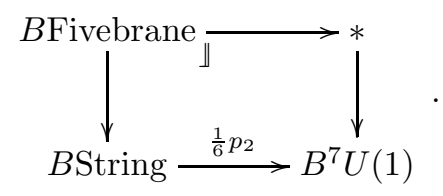

In direct analogy with def. 2.5 we therefore have the following notion.

Definition 2.16. For $X$ a manifold and $[c] \in H^{8}(X, \mathbb{Z})$ a class, we say that the space of $[c]$-twisted Fivebranestructures on $X$, denoted $\left(\frac{1}{6} p_{2}\right) \operatorname{Struc}_{[\alpha]}(X)$, is the homotopy pullback

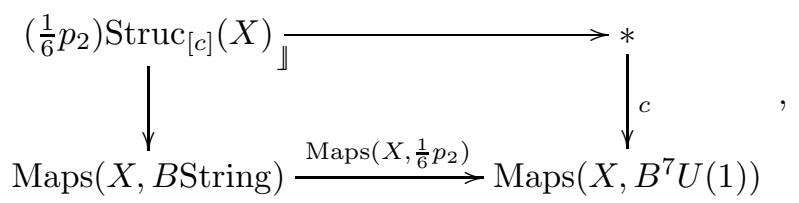

Explicitly, a $[c]$-twisted Fivebrane structure on a brane $\iota: Q \rightarrow X$ equipped with String structure $f: Q \rightarrow B$ String is a homotopy $\eta$ in a diagram analogous to (2.21)

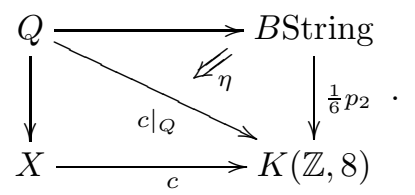

Two $[c]$-twisted Fivebrane structures $\eta$ and $\eta^{\prime}$ on $Q$ are regarded as equivalent if there is a homotopy between $\eta$ and $\eta^{\prime}$. In the case that $[c]=0$ this reduces to the untwisted Fivebrane structures considered in [47.

In terms of these notions we now have

Observation 2.17. For $X$ a manifold with String-structure and with a background gauge bundle $E \rightarrow X$ fixed such that $8 \operatorname{ch}(E)$ is integral, condition (2.22) is precisely the condition for the existence of $8 \operatorname{ch}(E)$ twisted Fivebrane-structure on $X$.

We now consider the above anomaly cancellation condition in more detail.

In [47] the main example of a Fivebrane structure came from the dual formulation [42] 25] of the GreenSchwarz anomaly cancellation mechanism [26, using the dual $H$-field $H_{7}$ of [14]. The expression is given by

$$
d H_{7}=2 \pi\left[\operatorname{ch}_{4}\left(F_{A}\right)-\frac{1}{48} p_{1}\left(F_{\omega}\right) \operatorname{ch}_{2}\left(F_{A}\right)+\frac{1}{64} p_{1}\left(F_{\omega}\right)^{2}-\frac{1}{48} p_{2}\left(F_{\omega}\right)\right],
$$

where $F_{A}$ and $F_{\omega}$ are the curvatures of the connections $A$ and $\omega$ on the gauge bundle $E$ and the tangent (or Spin) bundle of the ten-manifold $M$, respectively. In order to define a Fivebrane structure, we assume we already have a String structure, so we require $\frac{1}{2} p_{1}(T M)=0$. Then the expression (2.24) becomes

$$
d H_{7}=2 \pi\left[\operatorname{ch}_{4}\left(F_{A}\right)-\frac{1}{48} p_{2}\left(F_{\omega}\right)\right] .
$$


In 47. we had to find ways to get rid of the extra terms to isolate the non-decomposable terms. In the twisted formalism in this paper we see that the presence of such terms amounts to a piece of the twist and that it does not matter how many terms we have, as long as they are integral and have the same total degree and hence provide a map to $K(\mathbb{Z}, 8)$. Indeed, if we can define

$$
[\beta]:=-\operatorname{ch}_{4}(E): M \stackrel{!}{\longrightarrow} K(\mathbb{Z}, 8),
$$

i.e. require factorization

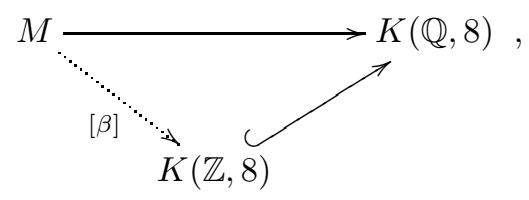

then we can reinterpret expression (2.25) as $\frac{1}{48} p_{2}(T M)+[\beta]=0$, since $\left[d H_{7}\right]=0$, the cohomology class of an exact form.

We discuss the validity of the map in (2.26). The Chern character is in general not an integral expression, but rather

$$
\operatorname{ch}: K^{0}(X) \rightarrow H^{\text {even }}(X ; \mathbb{Q}) .
$$

One way out of this is to first define a rational version of the twist, for which the map in (2.26) is replaced by a map from $M$ to the rational Eilenberg-MacLane space

$$
[\beta]:=-\operatorname{ch}_{4}(E): M \rightarrow K(\mathbb{Q}, 8),
$$

which gives that indeed $\operatorname{ch}_{4}(E)$ is in general in $[M, K(\mathbb{Q}, 8)]=H^{8}(M, \mathbb{Q})$. Hence

Definition 2.18. A rational Fivebrane twist on $M$ is a map from $M$ to $K(\mathbb{Q}, 8)$, i.e. an element of $H^{8}(M ; \mathbb{Q})$.

However, we can also give conditions under which the map in (2.26) is valid. The degree four Chern character is given by

$$
\mathrm{ch}_{4}=\frac{1}{24}\left(c_{1}^{4}-4 c_{1}^{2} c_{2}+4 c_{1} c_{3}+2 c_{2}^{2}-4 c_{4}\right) .
$$

The Chern classes are integral classes and so the Chern character is a priori integral up to a factor of 24 .

We describe this as follows. The Chern character is not integral in $B U$ but it will be integral in some lift, say $\mathcal{B U}$, of $B U$. Then we ask: when can we lift to this new space? This is given in terms of the following diagram

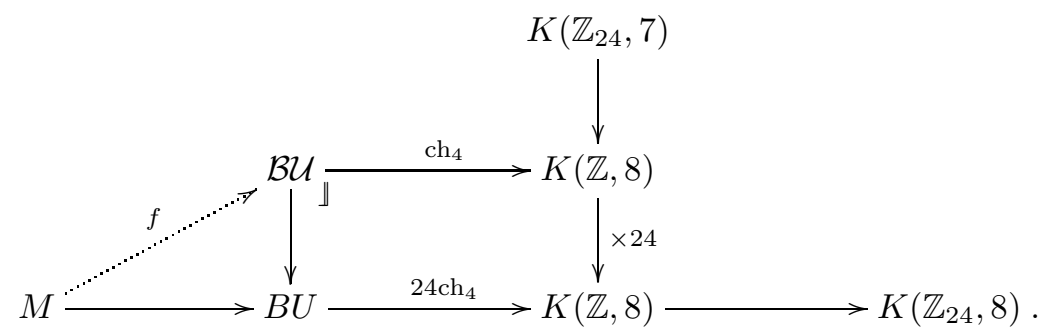

The right-most factor $K\left(\mathbb{Z}_{24}, 8\right)$ represents the obstruction: there is a class $k$ in $H^{8}\left(M ; \mathbb{Z}_{24}\right)$ which measures this obstruction. The top-most factor $K\left(\mathbb{Z}_{24}, 7\right)$ represents the different labeling of lifts $f$ to the new space $\mathcal{B U}$. If we take connected covers of $B U$ rather than $B U$ itself in the diagram, then we have that the space $\mathcal{B U}$ is isomorphic to another space in which $\frac{1}{6} c_{4}$, instead of $\mathrm{ch}_{4}$, is integral. The relevance of the unitary groups here is because they provide the adjoint representation for our structure groups and this is the representation relevant for Yang-Mills theory. For $E_{8}$, the adjoint representation is ad : $E_{8} \rightarrow S U(248)$, so that the adjoint representation of $G=E_{8} \times E_{8}$ is $(\mathrm{ad}, \mathrm{ad}): E_{8} \times E_{8} \rightarrow S U(248) \times S U(248) \hookrightarrow S U(496)$. 
Note that the above general discussion can be simplified. For both structure groups $E_{8} \times E_{8}$ and $\operatorname{Spin}(32) / \mathbb{Z}_{2}$ we have $c_{1}(E)=0$, so that for these groups $\operatorname{ch}_{4}(E)=\frac{1}{12}\left(c_{2}(E)^{2}-2 c_{4}(E)\right)$. We now consider the case when, in addition, we have $c_{2}(E)=0$. In this case, the formula for the Chern $\operatorname{character} \mathrm{ch}_{4}(E)$ further simplifies to

$$
\operatorname{ch}_{4}(E)=-\frac{1}{6} c_{4}(E)
$$

Here what we have really done is lifted the unitary group to its connected cover $B U\langle 8\rangle$. Indeed let us consider the result from [54] where the $\bmod p(p$ an odd prime) cohomology of the connective cover $B U\langle 2 n\rangle$ was calculated. From that result and the result of Stong [57] for $p=2$, the following divisibility result was deduced for all primes $p$ in $\left[54\right.$. Let $c_{k} \in H^{2 k}(B U ; \mathbb{Z})$ be the universal Chern class in $B U$, then the Chern class $r_{n}^{*}\left(c_{k}\right)$ in $B U\langle 2 n\rangle$ where $r_{n}: B U\langle 2 n\rangle \rightarrow B U$ be the canonical projection is divisible by [54]

$$
\prod_{p} p^{q}
$$

where $q$ is the least integer part of $\frac{(n-1)-\sigma_{p}(k-1)}{p-1}$, with $\sigma_{p}(n)=\sum a_{i}$ the sum of the coefficients in the unique decomposition of the integer $n$ as $n=a_{0}+a_{p}+\cdots+a_{k} p^{k}$, with $a_{i}<p$. Applying this result for $n=4$, $p=2,3$, and using $\sigma_{2}(3)=2, \sigma_{3}(3)=1$, we get that $r_{4}^{*}\left(c_{4}\right)$ is divisible by

$$
2^{\frac{2-\sigma_{2}(3)}{1}} \cdot 3^{\frac{3-\sigma_{3}(3)}{2}}=6 \text {. }
$$

We will give an example where this occurs and where the expression (2.32) is integral.

Example. Consider a complex vector bundle $E$ on the eight-sphere $S^{8}$. For ten-manifold we can simply take $S^{8} \times \mathbb{R}^{2}$ for example. The index of the Dirac operator on $S^{8}$ coupled to the vector bundle $E$ is given by the evaluation of the twisted $\widehat{A}$-genus $\widehat{A}\left(S^{8}, E\right):=\left(\operatorname{ch}(E) \cdot \widehat{A}\left(S^{8}\right)\right)\left[S^{8}\right]$ on the fundamental class $\left[S^{8}\right]$ of $S^{8}$

$$
\operatorname{Index} D_{E}=\left(\widehat{A}\left(T S^{8}\right) \cdot \operatorname{ch}(E)\right)=\operatorname{ch}(E)\left[S^{8}\right],
$$

as $\widehat{A}\left(T S^{8}\right)=1$, since spheres have stably trivial tangent bundles. Since $S^{8}$ is a Spin manifold, the index should be an integer. This then gives the requirement

$$
\operatorname{ch}_{4}(E)\left[S^{8}\right]=-\frac{1}{6} c_{4}(E)\left[S^{8}\right] \in \mathbb{Z} .
$$

2.3.2. The M5-brane and the dual $C$-field. The magnetic dual of the M2-brane is the M5-brane. Where the M2-brane is electrically charged under the $C_{3}$-field with class $\left[G_{4}\right] \in H^{4}(X, \mathbb{Z})$, the M5-brane is electrically charged under the dual $C_{6}$-field with class $\left[G_{8}\right] \in H^{8}(X, \mathbb{Z})$. If $X$ admits a String-structure, then, as we discuss in more detail in a moment, one finds an anomaly cancellation condition on these background fields analogous to (2.15) which reads

$$
8\left[G_{8}\right]=4 a(E) \cup a(E)-\frac{1}{6} p_{2}(T X),
$$

where $a: B E_{8} \rightarrow K(\mathbb{Z}, 4)$ is a representative of the canonical degree- 4 class on the classifying space of the exceptional Lie group $E_{8}$.

The homotopy-theoretic interpretation of this condition involves the following Fivebrane-analog of Spin ${ }^{c}$ as it appeared in prop. 2.7, and of String $^{\alpha}$ as it was considered in def. 2.8.

Definition 2.19. For $G$ a topological group and $[\alpha] \in H^{8}(B G, \mathbb{Z})$ a universal 8-class, we say that Fivebrane ${ }^{\alpha}$ is the loop group of the homotopy pullback

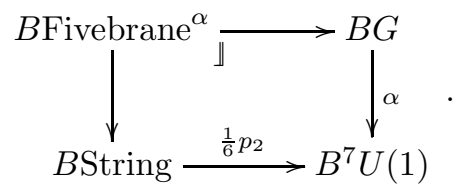


As before with String $^{\alpha}$, this notion in particular subsumes spaces on which the class $\frac{1}{6} p_{2}$ is further divisble. For let $G=B^{6} U(1)$ in the above and take $\alpha:=\mathrm{DD}_{6}$ to be the canonical degree- 8 class on $B^{7} U(1) \simeq K(\mathbb{Z}, 8)$. This is the canonical class of circle 7-bundles / U(1)-bundle 6-gerbes. Then Fivebrane ${ }^{8 D_{6}}$ is the loop group of the homotopy pullback

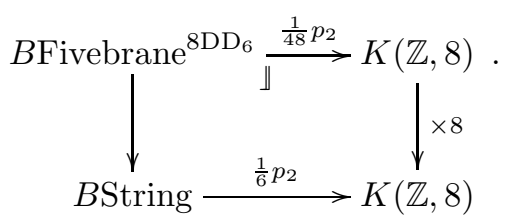

Accordingly, we have that a String-bundle lifts to a Fivebrane ${ }^{8 D_{6}}$-bundle precisely if the class of $\frac{1}{6} p_{2}$ is further divisible by 8 , hence if $\frac{1}{48} p_{2}$ exists. In [47] this space was denoted

$$
\mathcal{F}^{\langle 8\rangle}:=B \text { Fivebrane }{ }^{8 \mathrm{DD}_{6}} .
$$

Moreover, the class $\frac{1}{48} p_{2} \in H^{8}$ (Fivebrane $\left.{ }^{8 D_{6}} ; \mathbb{Z}\right)$ is the universal obstruction to lifting to a genuine Fivebranebundle. Accordingly, we have a notion of twisted Fivebrane-structures induced by $\frac{1}{48} p_{2}$. They form the space given by the homotopy pullback.

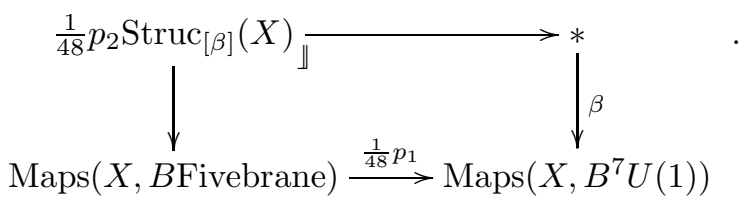

Such a $[\beta]$-twisted Fivebrane-structure on a brane $\iota: Q \hookrightarrow X$ is a homotopy $\eta$ in the diagram

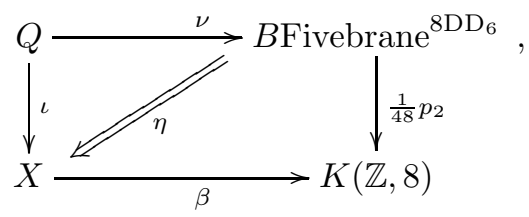

which exists precisely if

$$
\frac{1}{48} p_{2}(X)+\iota^{*}([\beta])=0 .
$$

We may also regard the situation in analogy with def. 2.10 and consider the following.

Definition 2.20. For $X$ a manifold and for $[c] \in H^{8}(X ; \mathbb{Z})$ a degree 8 cohomology class, the space $\left(\frac{1}{6} p_{2}-2 a \cup 2 a\right) \operatorname{Struc}_{[c]}(X)$ of $[c]$-twisted Fivebrane ${ }^{2 a \cup 2 a}$-structures on $X$ is the homotopy pullback

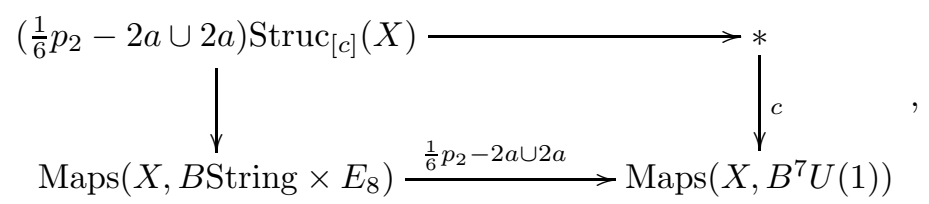

where the right vertical map picks a cocycle $c$ representing the class $[c]$.

In terms of these notions we thus see that

Observation 2.21. Over a manifold $X$ with String-structure and with a fixed gauge bundle $E$, condition (2.37) is precisely the condition that guarantees existence of a lift to $\left[8 G_{8}\right]$-twisted Fivebrane ${ }^{2 a \cup 2 a}$-structure through the left vertical morphism in def. 2.20. 
We discuss now how (2.37) arises in more detail. Locally the $C_{3}$-field is given by a 3 -form, which is traditionally denoted by the same symbol. The equation of motion for $C_{3}$ is obtained from varying the action

$$
S\left(C_{3}\right)=\int_{Y}\left[G_{4} \wedge * G_{4}+\frac{1}{6} G_{4} \wedge G_{4} \wedge C_{3}-I_{8} \wedge C_{3}\right]
$$

on an eleven-dimesional Spin manifold $Y$ to obtain

$$
d * G_{4}=-\frac{1}{2} G_{4} \wedge G_{4}+I_{8}
$$

Here $I_{8}$ is the one-loop polynomial [59] [19] given in terms of the Pontrjagin classes of the tangent bundle $T Y$ to $Y$

$$
I_{8}=\frac{p_{2}(T Y)-\frac{1}{2}\left(\frac{1}{2} p_{1}(T Y)\right)^{2}}{48}
$$

and $*$ is the Hodge duality operator for a given metric in eleven dimensions.

The integral lift of (2.44) leads to a class defined in [16]

$$
\begin{aligned}
{\left[G_{8}\right] } & =\left[\frac{1}{2} G_{4}^{2}-I_{8}\right] \\
& =\frac{1}{2} a(a-\lambda)+\frac{7 \lambda^{2}-p_{2}}{48}
\end{aligned}
$$

where $\lambda=\frac{1}{2} p_{1}$, and $a$ is the degree four class of an $E_{8}$ bundle coming from Witten's shifted quantization condition for $G_{4} 63$

$$
\left[G_{4}\right]=a-\frac{1}{2} \lambda=a-\frac{1}{4} p_{1}
$$

In [66] Witten interpreted the vanishing of a certain torsion class $\theta$ on the M-fivebrane worldvolume as a necessary condition for the decoupling of the fivebrane from the ambient space ( "the bulk"). Hence the vanishing of $\theta$ meant that the fivebrane can have a well-defined partition function. Consider the embedding $\iota: W \hookrightarrow Y$ of the fivebrane with six-dimensional worldvolume $W$ into eleven-dimensional spacetime $Y$. Consider the ten-dimensional unit sphere bundle $\pi: X \rightarrow W$ of $W$ with fiber $S^{4}$ associated to the normal bundle $N \rightarrow W$ of the embedding $\iota$. Then it was shown in [16] that the integration of $G_{8}$ over the fiber of $X$ gives exactly the torsion class $\theta$ on the fivebrane worldvolume

$$
\theta=\pi_{*}\left(G_{8}\right) \in H^{4}(W ; \mathbb{Z})
$$

Therefore, the vanishing of $G_{8}$ is a necessary condition for the existence of a non-zero partition function [16].

We now proceed with the interpretation. Since we have Fivebrane structures in mind, we assume that $Y$ already admits a String structure, i.e. that $\frac{1}{2} p_{1}(Y)=0$. Then, from (2.46) we see that the class $G_{8}(Y)$ simplifies to

$$
G_{8}(Y)=\frac{1}{2} a^{2}-\frac{1}{48} p_{2}(Y) .
$$

The class $a$ is an integral class of an $E_{8}$ bundle and hence defines a map to $K(\mathbb{Z}, 4)$. Then the square of $a$ defines a map to $K(\mathbb{Z}, 8)$, and hence defines a twist for us. As we also have the class $\frac{1}{48} p_{2}$, then we have a twist for the modified Fivebrane structure.

Necessity of the Fivebrane condition? The Fivebrane condition is stronger than simply the requirement that the one-loop term $I_{8}$ to vanish. For the former we require the obstructions $\frac{1}{2} p_{1}$ and $\frac{1}{6} p_{2}$ vanish separately, whereas for the latter we only require the combination to vanish. This has been studied in [31] 30] 62]. For instance, following 62, a Riemannian 8-dimensional spin manifold $M^{8}$ is said to be doubly supersymmetric if and only if the tangent bundle $T M^{8}$ and the spinor bundles $\Delta_{+} M^{8}$ and $\Delta_{-} M^{8}$ are associated with a principal 
$G$-fiber bundle such that there exist $G$-invariant isomorphisms between any two of the three bundles, i.e. $T M^{8}=\Delta_{+} M^{8}=\Delta_{-} M^{8}$. If $M^{8}$ is doubly supersymmetric,

$$
w_{1}=w_{2}=0, \quad e=0, \quad 4 p_{2}=p_{1}^{2},
$$

where $e$ is the Euler class. Then this implies for the signature $\operatorname{sgn}\left(M^{8}\right)=16 \hat{A}\left[M^{8}\right]$. In particular, $\operatorname{sgn}\left(M^{8}\right) \equiv$ 0 mod 16. One example is $P S U(3)$-structure for which

$$
\begin{aligned}
w_{i} & =0(i \neq 4), & & w_{4}^{2}=0 \\
e & =0, & & p_{1}^{2}=4 p_{2} .
\end{aligned}
$$

In particular, all Stiefel-Whitney numbers vanish.

A second example is a differentiable 8 -fold $M^{8}$ with an odd topological generalized Spin(7)-structure (in the sense of [62]) for which

$$
\chi\left(M^{8}\right)=0, \quad p_{1}\left(M^{8}\right)^{2}-4 p_{2}\left(M^{8}\right)=0 .
$$

The 7-sphere admits a Spin structure and therefore admits a generalized $G_{2}$-structure. The tangent bundle of the 8-sphere is stably trivial and therefore all the Pontrjagin classes vanish. Since the Euler class is non-trivial, there exists no generalized Spin(7)-structure on an 8-sphere. However, equation (2.53) is automatically satisfied for manifolds of the form $M^{8}=S^{1} \times N^{7}$ with $N^{7}$ Spin.

\section{Twisted Differential Structures in String Theory}

We now indicate a theory of nonabelian differential cohomology in which the topological structures considered in section 2 have smooth and differential refinements. A full account of this theory is given in [49]. The complete differential refinements are based on a smooth refinement of topological spaces by structures called higher smooth stacks or higher smooth groupoids. The full constructions of these, for the cases considered here, are discussed in [21. As shown there, underlying any such differential cohomological structure is, locally, explicit differential geometric data, given by differential forms with values in $L_{\infty}$-algebras. This $L_{\infty}$-algebra valued connection data by itself was discussed in [46], a brief collection of relevant $L_{\infty}$-algebraic notions is in the appendix section $\mathrm{A}$

Here, after a brief survey of the general theory, we concentrate on a discussion of this local differential form data for the differential refinements of the twisted String- and Fivebrane-structures from section 2 We show that these reproduce the equations on differential forms that are traditional in the physics literature on the Green-Schwarz anomaly cancellation as well as its magnetic dual.

The key fact is the existence of definition 3.2 below, which gives smooth and differential refinements of the homotopy pullbacks that defined twisted String- and Fivebrane-structures in def. 2.5 and def. 2.16] respectively.

3.1. Differential twisted cohomology. Differential twisted cohomology is the pairing of the notions of twisted cohomology with differential cohomology (see [28]). We have that

- a cocycle in differential cohomology is to the underlying bare cocycle as a connection on an $\infty$-bundle is to the underlying principal $\infty$-bundle;

- a cocycle in twisted cohomology is to an ordinary cocycle as a twisted bundle is to a principal bundle.

We indicate now briefly a formal definition of such objects as described in full detail in [49, showing how they connect via the constructions from 21] the cohomological discussion that we had so far to the $L_{\infty}$-algebraic differential form data of [46] in terms of which we shall obtain the relevant twisted Bianchi identities.

The basic mechanism is to refine the homotopy theory by passing from the $\infty$-topos [37. Top $\simeq \infty$ Grpd of topological spaces - equivalently: geometrically discrete $\infty$-groupoids - to that of smooth $\infty$-groupoids, defined as the $\infty$-category of $\infty$-stacks over the site of smooth manifolds.

It is a familiar fact that Lie groupoids, such as for instance orbifolds, are naturally to be thought of as stacks on the site of smooth manifolds, often called differentiable stacks [7. Such differentiable stacks form a common generalization of smooth manifolds and a small fragment of homotopy theory. For instance, for 
each Lie group $G$ there is a Lie groupoid, denoted $* / / G$ or $\mathbf{B} G$, with a single object and $G$ as its space of morphisms, such that for $X$ any smooth manifold, regarded as a Lie groupoid, the collection $\mathbf{H}(X, \mathbf{B} G)$ of stack morphisms $X \rightarrow \mathbf{B} G$ forms the groupoid of smooth $G$-principal bundles on $X$ and smooth gauge transformations between them. So the Lie groupoid $\mathbf{B} G$ serves as a smooth refinement of the topological classifying space $B G$.

But in the context of ordinary stacks, not all the classifying spaces that we considered in the previous section, such as $B^{n} U(1) \simeq K(\mathbb{Z}, n+1)$ for higher $n$, have a smooth refinement to stacks on manifolds. But there is a natural generalization of the theory of stacks to a general theory of higher stacks, often called $\infty$-stacks. The collection of all of these over a given site is an $\infty$-topos [37] and this notion serves as a complete joint unification of the geometry over the given site with homotopy theory.

For instance, over the trivial site an $\infty$-stack is the same as an $\infty$-groupoid (a Kan complex), which in turn is equivalently - in the sense of homotopy theory - a topological space. We say that the collection of all of these

$$
\text { Top } \simeq \infty \mathrm{Grpd}
$$

is the canonical base $\infty$-topos. Next, in generalization to the relation between Lie groupoids and differentiable stacks, we speak of the $\infty$-stacks on the site of smooth manifolds as being smooth $\infty$-groupoids. We write $\mathbf{H}:=$ Smooth $\infty$ Grpd for the $\infty$-topos of smooth $\infty$-groupoids. For emphasis, we will say that an object in $\infty$ Grpd is a bare or discrete $\infty$-groupoid (not equipped with nontrivial smooth structure). Notice that therefore also topological spaces are, in the sense of homotopy theory, discrete $\infty$-groupoids.

It turns out that the $\infty$-topos Smooth $\infty$ Grpd sits over that of bare $\infty$-groupoids by an adjoint quadruple of $\infty$-functors

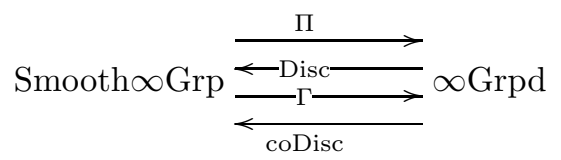

and this controls the notion of smooth refinement of bare cohomology. The geometric interpretation is this:

- "Disc" produces smooth $\infty$-groupoids with discrete smooth structure;

- " $\Gamma$ " forgets the smooth structure on a smooth $\infty$-groupoid;

- " $\Pi$ " sends a smooth $\infty$-groupoid $X$ to its fundamental path $\infty$-groupoid; combined with the equivalence $\infty \mathrm{Grpd} \simeq$ Top this is the operation of geometric realization.

For any object or diagram in $\infty$ Grpd by a smooth lift of it we mean a lift through $\Pi$ to an object or diagram, respectively, in Smooth $\infty$ Grpd.

A smooth $\infty$-groupoid with a single object we write $\mathbf{B} G$, where $G$ is the smooth $\infty$-group of automorphisms of that single object. The boldface $\mathbf{B}$ denotes delooping in Smooth $\infty$ Grpd as opposed to in $\infty$ Grpd. This completely defines both structures: pointed connected smooth $\infty$-groupoids are equivalent to smooth $\infty$ groups. For instance there is for each $n \in \mathbb{N}$ a smooth $\infty$-groupoid $\mathbf{B}^{n} U(1)$, defined this way inductively from the ordinary smooth circle group $U(1)=\mathbf{B}^{0} U(1)$. This is such that $\Pi \mathbf{B}^{n} U(1) \simeq B^{n+1} \mathbb{Z} \simeq K(\mathbb{Z}, n+1)$ is the Eilenberg-MacLane space that classifies integral cohomology in degree $n$. Hence $\mathbf{B}^{n} U(1)$ is a smooth refinement of the classifying space $B^{n} U(1) \simeq K(\mathbb{Z}, n+1)$.

A morphism $X \rightarrow \mathbf{B}^{n} U(1)$ of smooth $\infty$-groupoids classifies a circle $n$-bundle on $X$. For $n=1$ and $X$ an ordinary manifold this are ordinary circle bundle, for $n=2$ this are bundle gerbes, for $n=3$ this are bundle 2 -gerbes, etc. Generally, a morphism $X \rightarrow \mathbf{B} G$ classifies a $G$-principal $\infty$-bundle.

Note that $X$ here can be much more general than a smooth manifold. Notably, we can have $X=\mathbf{B} G$ for $G$ a Lie group or more general smooth $\infty$-group. A morphisms $\mathbf{c}: \mathbf{B} G \rightarrow \mathbf{B}^{n} U(1)$ then defines a cocycle in generalized (Segal-Brylinski-)smooth group cohomology on $G$ with coefficients in $U(1)$ in degree $n$. If $G$ is a Lie group or an $\infty$-group presented by a simplicial Lie group, then $\Pi \mathbf{B} G \simeq B G$ is the ordinary classifying space of $G$. Therefore such a cocycle maps under $\Pi$ to an ordinary integral cocycle

$$
\left[\Pi\left(\mathbf{B} G \stackrel{\mathbf{c}}{\rightarrow} \mathbf{B}^{n} U(1)\right)\right] \simeq[B G \stackrel{c}{\rightarrow} K(\mathbb{Z}, n+1)] \in H^{n+1}(B G, \mathbb{Z})
$$


on the classifying space. So the cocycle $\mathbf{c}$ is a smooth refinement of the topological characteristic map $c$. We say that $\mathbf{c}$ lives in the smooth cohomology $H_{\text {smooth }}^{n}(\mathbf{B} G, U(1))$.

Given any such cocycle $\mathbf{c}$, we say that the c-twisted cohomology on $X$ is the connected components of the homotopy pullback

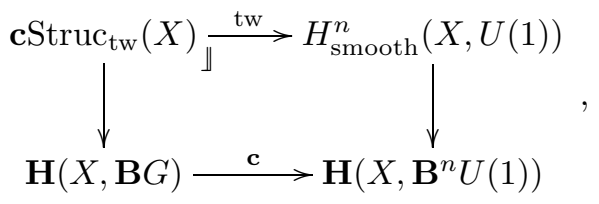

where the right vertical morphism is the canonical effective epimorphism that picks one cocycle in each cohomology class. The map tw here sends twisted cocycles to their twist. For instance for $\mathbf{c}: \mathbf{B} P U(n) \rightarrow$ $\mathbf{B}^{2} U(1)$ the cocycle that classifies the extension $\mathbf{B} U(1) \rightarrow \mathbf{B} U(n) \rightarrow \mathbf{B} P U(n)$ we have that $\mathbf{c S t r u c}_{\mathrm{tw}}(X)$ is the groupoid of twisted complex vector bundles of rank $n$ on $X$, those that appear in the geometric model for twisted K-theory in degree 0.

The reader may be more familiar with twisted cohomology formulated in terms of sections of certain bundles. We briefly indicate how this is equivalently another perspective on the above setup.

Consider first the example of a Lie group $G$ acting on a vector space $V$. The weak quotient of this action is a Lie groupoid $V / / G$ whose objects form the space $V$ and where there is precisely one morphism for every ordered pair of points related by the group action. This Lie groupoid is equipped with a cononical projection $\rho: V / / G \rightarrow \mathbf{B} G$. We may think of this as the smooth incarnation of the vector bundle that is associated via $\rho$ to the universal $G$-bundle over $\mathbf{B} G$. In fact we have a fiber sequence $V \rightarrow V / / G \stackrel{\rho}{\rightarrow} \mathbf{B} G$ of Lie groupoids, and this may equivalently be taken to define the action $\rho$ of $G$ on $V$.

Now consider a morphism $g: X \rightarrow \mathbf{B} G$ classifying a $G$-principal bundle $P \rightarrow X$, as above. By inspection one finds that a lift $\sigma$ of this morphism along this projection

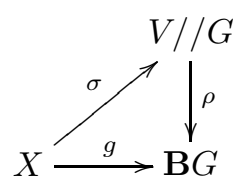

is precisely a section of the vector bundle $P \times_{\rho} V$ that is associated to $P$ by the given representation. On the other hand, in terms of the above notion of twisted cohomology, such a lift is also precisley an element in the $\rho$-twisted cohomology of $X$ with coefficients in $V / / G$, where the twist is the class of $P$ : we have an equivalence

$$
\operatorname{Struc}_{[P]}(X) \simeq \Gamma_{X}\left(P \times_{\rho} V\right)
$$

of cocycles in $\rho$-twisted cohomology with sections of the $\rho$-associated vector bundle.

This perspective generalizes verbatim to all twisting cocycles $\mathbf{c}$ on all $\infty$-groups. For those of the form $\mathbf{c}: \mathbf{B} G \rightarrow \mathbf{B}^{n} U(1)$ considered before, we may think of their homotopy fiber $\mathbf{B} \hat{G}$ in the fiber sequence

$$
\mathbf{B} \hat{G} \rightarrow \mathbf{B} G \stackrel{\mathbf{c}}{\rightarrow} \mathbf{B}^{n} U(1)
$$

as the delooping of the shifted central extension $\hat{G}$ of $G$ classified by the cocycle $\mathbf{c}$, or equivalently think of $\mathbf{B} G$ as a universal c-associated $\mathbf{B} \hat{G}$-bundle over $\mathbf{B}^{n} U(1)$. Then for $P \rightarrow X$ classified by $g: X \rightarrow \mathbf{B}^{n} U(1)$ a given circle $n$-bundle on $X$, the $\infty$-groupoid $\mathbf{c S t r u c}_{[g]}$ may be thought of equivalently as the space of sections of the associated $\mathbf{B} \hat{G}$-bundle. If $P$ is trivial, such sections are just maps from $X$ to $\mathbf{B} \hat{G}$.

In order to equip such structures of smooth and twisted cohomology with connections, we reflect $\Pi$ back to smooth $\infty$-groupoids by defining $\Pi:=$ Disc $\Pi$ : Smooth $\infty$ Grpd $\rightarrow$ Smooth $\infty$ Grpd. For $X$ a smooth $\infty$ groupoid we say that $\Pi(X)$ is its smooth path $\infty$-groupoid. The adjunction unit gives a canonical morphism $X \rightarrow \Pi(X)$ which includes $X$ as the constant paths in $X$. A morphism $\boldsymbol{\Pi}(X) \rightarrow \mathbf{B} G$ therefore has an underlying $G$-principal $\infty$-bundle $X \rightarrow \Pi(X) \rightarrow \mathbf{B} G$ but also assigns equivalences between fibers over 
endpoints of paths, that are equivalent around disks, etc. This is the parallel transport of a flat $\infty$-connection on the smooth $G$-principal bundle.

From this in turn derives a notion of $G$-valued flat differential forms: these are the flat $G$-connections whose underlying $\infty$-bundle is trivial. We write

$$
\Pi_{\mathrm{dR}} X:=\Pi X \coprod_{X} *
$$

for the canonical homotopy pushout and speak of the de Rham homotopy type of $X$. A morphism $\boldsymbol{\Pi}_{\mathrm{dR}}(X) \rightarrow$ $\mathbf{B} G$ is a closed $G$-valued form on $X$. In particular a morphism $\boldsymbol{\Pi}_{\mathrm{dR}}(X) \rightarrow \mathbf{B}^{n} U(1)$ is a closed $n$-form.

A key observation now is that there is a canonically induced morphism of cocycle $\infty$-groupoids

$$
\text { curv : } \mathbf{H}\left(X, \mathbf{B}^{n} U(1)\right) \rightarrow \mathbf{H}\left(\mathbf{\Pi}_{\mathrm{dR}}(X), \mathbf{B}^{n+1} U(1)\right)
$$

that sends each circle $n$-bundle to a curvature characteristic form. We define ordinary differential cohomology $\mathbf{H}_{\text {diff }}\left(X, \mathbf{B}^{n} U(1)\right)$ of $X$ to be the $\infty$-pullback of the canonical effective epimorphism $H_{\mathrm{dR}}^{n}(X) \rightarrow$ Smooth $\infty \operatorname{Grpd}\left(\boldsymbol{\Pi}_{\mathrm{dR}}(X), \mathbf{B}^{n+1} U(1)\right)$ (that which picks one cocycle in each cohomology class) along this curvature characteristic map. Cocyles in this homotopy pullback

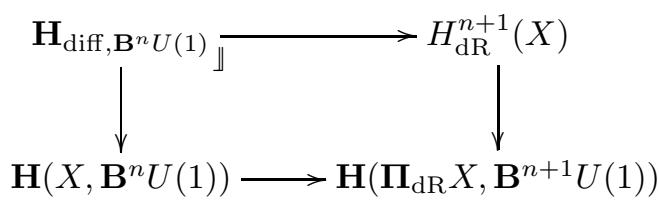

are smooth circle $n$-bundles with connection.

For $X$ an ordinary manifold, this reproduces the ordinary notions in differential cohomology. More precisely, in this case the $n$-groupoid $\mathbf{H}_{\text {diff }}\left(X, \mathbf{B}^{n} U(1)\right)$ turns out to be that whose objects are cocycles in Deligne-Beilinson hypercohomology, whose morphisms are smooth gauge transformations of these, whose 2-morphisms are higher gauge transformations of those, and so on. But as before, we can apply this over any smooth $\infty$-groupoid. In particular we may consider differential cohomology over moduli stacks $\mathbf{B} G$ of $G$-principal $\infty$-bundles that differentially refine smooth lifts $\mathbf{c}: \mathbf{B} G \rightarrow \mathbf{B}^{n+1} U(1)$ of characteristic map. These are Chern-Simons n-gerbes with connection.

This finally gives rise to the notion of $\infty$-connections on general nonabelian $G$-principal $\infty$-bundles: these are structures that lift universal curvature classes from de Rham cohomology to differential cohomology.

With all these concepts thus abstractly given, we can look for explicit constructions of these. By standard theory [37] every smooth $\infty$-groupoid $A$ is presented a simplicial presheaf on the category of Cartesian spaces and smooth maps: a functor

$$
A:\left(U=\mathbb{R}^{n},[k]\right) \mapsto A_{k}(U) \in \text { Set }
$$

that we read as assigning to each test space $U$ and each $k \in \mathbb{N}$ the set of $k$-morphisms of the $\infty$-groupoid of possible ways of probing $A$ with $U$, or equivalently the set of $U$-parameterized smooth families of $k$-morphisms in $A$.

We consider the construction of such simplicial presheaves from infinitesimal data. For $\mathfrak{g}$ an an $L_{\infty^{-}}$ algebra (see [46] and the appendix for a review or relevant notions) we can define such a presheaf by setting, in evident generalization of the construction in [27,

$$
\exp (\mathfrak{g}):(U,[k]) \mapsto\left\{\Omega^{\bullet}\left(U \times \Delta^{k}\right)_{\text {vert }} \stackrel{A_{\text {vert }}}{\longleftarrow} \mathrm{CE}(\mathfrak{g})\right\},
$$

where on the right we have the set of dg-algebra homomorphisms from the Chevalley-Eilenberg algebra of $\mathfrak{g}$ to the de Rham complex of vertical forms on the trivial simplex bundle $U \times \Delta^{k} \rightarrow U$. The Chevalley-Eilenberg dg-algebra $\mathrm{CE}(\mathfrak{g})$ is the free graded-commutative algebra on the the degreewise dual of the graded vector space underlying $\mathfrak{g}$ and equipped with the differential obtained by dualizing all the brackets on $\mathfrak{g}$. For $\mathfrak{g}$ an ordinary Lie algebra it reduces to the ordinary Chevalley-Eilenberg algebra, hence its name.

This simplicial presheaf $\exp (\mathfrak{g})$ presents the smooth $\infty$-groupoid $\mathbf{B} G$ for $G$ the "universal $\infty$-connected" Lie integration of $\mathfrak{g}$. Smooth Postnikov truncations $\tau_{n}$ of this object yield smooth $n$-groups integrating $\mathfrak{g}$. 
For instance for $\mathfrak{g}$ an ordinary Lie algebra we have that $\tau_{1} \exp (\mathfrak{g}) \simeq \mathbf{B} G$ for $G$ the ordinary simply connected Lie group integrating $\mathfrak{g}$.

The crucial point for our discussion here is that one obtains from this also a model for the smooth $\infty$ groupoid that classifies $G$-principal $\infty$-connections for such Lie integrated $G$ [21]. If we write $\mathrm{W}(\mathfrak{g})$ for the Weil algebra of $\mathfrak{g}$, the unique free dg-algebra on the dual graded vector space of $\mathfrak{g}$ such that the canonical projection is a dg-algebra homomorphism to $\mathrm{CE}(\mathfrak{g})$, and $\operatorname{inv}(\mathfrak{g})$ for the subalgebra of closed elements formed out of shifted generators - the invariant polynomials on $\mathfrak{g}-$, then this is given by the simplicial presheaf defined by

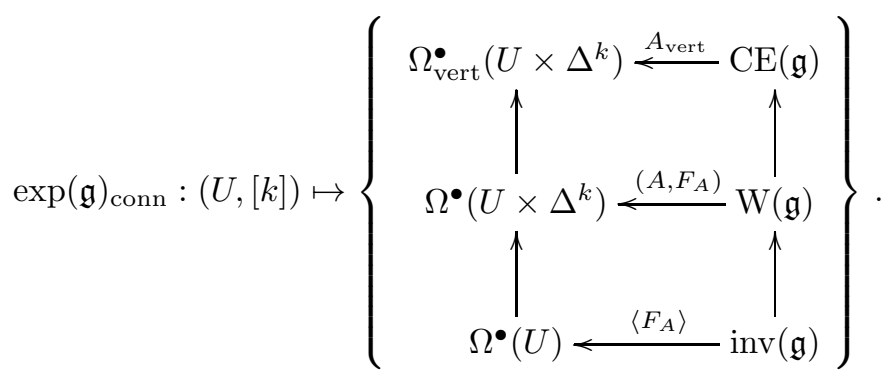

On the right we have the set of horizontal dg-algebra homomorphisms that makes the diagram commute, see around def. A.7 in the appendix for more details. This is the $L_{\infty}$-algebraic differential form data discussed in detail in [46], here parameterized over all test spaces $U$ and simplices $\Delta^{k}$ as discussed in [21. The horizontal morphism in the middle constitutes $\mathfrak{g}$-valued differential form data on $U \times \Delta^{k}$ and the fact that it sits in this commuting diagram encodes the $\infty$-analogs of the two conditions of an ordinary Cartan-Ehresmann connection: the top square says that the vertical part of $A$ is flat, and the bottom square says that the curvature forms "transform covariantly" and make all invariant polynomials descent down to $U$.

For $X$ a smooth manifold a morphism $X \rightarrow \exp (\mathfrak{g})_{\text {conn }}$ is equivalently

(1) a choice of good open cover $\left\{U_{i} \rightarrow X\right\}$;

(2) on each patch $U_{i}$, differential form data with values in $\mathfrak{g}$;

(3) on each double intersection, a choice of 1-parameter gauge transformation between the corresponding differential form data;

(4) on each triple intersection, a choice of 2-parameter gauge-of-gauge transformation;

(5) and so on for higher intersections.

Given then a differential refinement of a cocycle $\mathbf{c}: \mathbf{B} G \rightarrow \mathbf{B}^{n} U(1)$ to the corresponding moduli stacks of bundles with connection

$$
\hat{\mathbf{c}}: \mathbf{B} G_{\text {conn }} \rightarrow \mathbf{B}^{n} U(1)_{\text {conn }},
$$

we can consider the differential refinement of the twisted smooth cohomology discussed above: the cocycle $\infty$-groupoid of $\hat{\mathbf{c}}$-twisted differential cohomology is the homotopy pullback

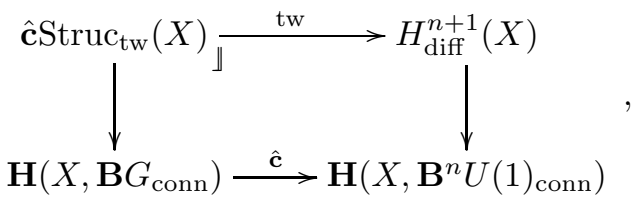

where again the right vertical morphism is the canonical effective epimorphism that picks one cocycle in each cohomology class. Up to some slight technicalities which are discussed in 21, this homotopy pullback is modeled by the corresponding pullback of the double square diagrams of $L_{\infty}$-algebra data from above, which are discussed in detail in the last part of [46. This we will later unwind in sections 3.2 and 3.3 for the case of twisted differential String- and Fivebrane structures. 
Facts 3.1. (1) There is, up to equivalence, a unique smooth refinement of the first fractional Pontrjagin class (from fact 2.3 )

$$
\frac{1}{2} \mathbf{p}_{1}: \text { BSpin } \rightarrow \mathbf{B}^{3} U(1)
$$

to the moduli stack of smooth Spin-principal bundles with values in the moduli 3-stack of circle 3-bundle.

(2) The smooth string 2-group, whose smooth delooping we denote BString, is the homotopy fiber of $\frac{1}{2} \mathbf{p}_{1}$, sitting in a fiber sequence

$$
\text { BString } \rightarrow \text { BSpin } \stackrel{\frac{1}{2} \mathbf{p}_{1}}{\longrightarrow} \mathbf{B}^{3} U(1) .
$$

(3) The models for the smooth string 2-group given in [27] and in [4] are indeed presentations of the abstract definition (3.15).

(4) There is a smooth refinement $\frac{1}{6} \mathbf{p}_{2}: \mathbf{B S t r i n g} \rightarrow \mathbf{B}^{7} U(1)$ of the second fractional Pontrjagin class from fact 2.14 to the moduli 2-stack of String-principal 2-bundles with values in the moduli 7-stack of circle 7-bundles.

(5) The homotopy fiber of $\frac{1}{2} \mathbf{p}_{2}$ is the smooth delooping of the smooth fivebrane 6-group

$$
\text { BFivebrane } \rightarrow \text { BString } \stackrel{\frac{1}{6} \mathbf{p}_{2}}{\longrightarrow} \mathbf{B}^{7} U(1) .
$$

(6) Under geometric realization these smooth lifts indeed reproduce the first steps in the Whitehead of $O$ :

$$
\mid \mathbf{B} \text { String } \mid \simeq B \text { String } \quad \text { and } \quad|\mathbf{B F i v e b r a n e}| \simeq B \text { Fivebrane } .
$$

This is shown in 21] and also discussed in section 4.1 of [49].

In summary all this means that we obtain the following canonical refinement of def. 2.5$]$ and def. 2.16 to a notion of twisted differential string- and fivebrane structures.

Definition 3.2. For $X$ a smooth manifold, the 2-groupoid of twisted differential String-structures $\frac{1}{2} \hat{\mathbf{p}}_{1} \operatorname{Struc}_{\mathrm{tw}}(X)$ on $X$ is the homotopy pullback

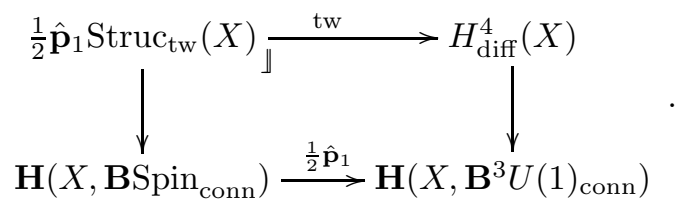

Analogously, the 6-groupoid of twisted differential fivebrane-structures $\frac{1}{6} \hat{\mathbf{p}}_{2} \operatorname{Struc}_{\mathrm{tw}}(X)$ on $X$ is the homotopy pullback

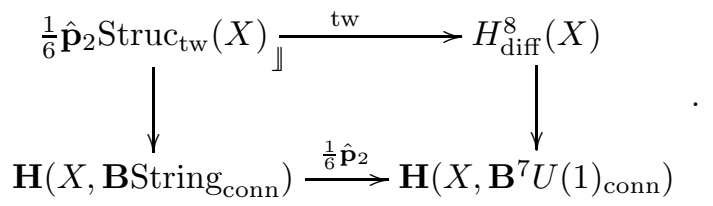

Notice that since these constructions had been announced in [46] the article 60 has appeared which defines a presentation of twisted differental String-structures in terms of bundle 2-gerbes for the case that the underlying topological twist vanishes.

We now describe the local $L_{\infty}$-algebraic differential form data of such twisted differential structures, following [46]. Details of the following fact are in [21].

Theorem 3.3. (i) The local differential form data of a twisted String $(n)$-bundle with connection is that known from the Green-Schwarz mechanism (section 2.2.1).

(ii) The local differential form data of a twisted Fivebrane(n)-bundle with connection is that of the dual Green-Schwarz mechanism (section 2.3.1). 
3.2. Twisted $\mathfrak{s t r i n g}(n)$ 2-connections. One finds that the smooth first fractional Pontryagin map

$$
\frac{1}{2} p_{1}: \mathbf{B S p i n}(n) \rightarrow \mathbf{B}^{3}(U(1))
$$

from the moduli stack of Spin-principal bndles to the moduli 3-stack of circle 3-bundles may be modeled in terms of simplicial presheaves by the span of smooth 2-groupoids of the form

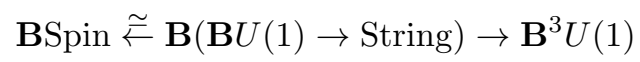

given by a span of smooth crossed complexes of the form

$$
(1 \longrightarrow 1 \longrightarrow \operatorname{Spin}(n)) \stackrel{\simeq}{\longleftarrow}(U(1) \longrightarrow \hat{\Omega} \operatorname{Spin}(n) \longrightarrow P \operatorname{Spin}(n)) \stackrel{\frac{1}{2} p_{1}}{\longrightarrow}(U(1) \longrightarrow 1 \longrightarrow 1)
$$

where $\hat{\Omega}$ Spin denotes the Kac-Moody central extension of the loop group of Spin.

The corresponding span of $L_{\infty}$-algebras is of the form

$$
\mathfrak{s o}(n) \stackrel{\simeq}{\longleftarrow}(b \mathfrak{u}(1) \rightarrow \mathfrak{s t r i n g}(n)) \rightarrow b^{2} u(1),
$$

where in the middle we we have the mapping cone of the inclusion of the line Lie 2-algebra, example A.2 into the string Lie 2-algebra, example A.5.

Therefore for $C\left(\left\{U_{i}\right\}\right) \stackrel{\widetilde{ }}{\rightarrow} X$ the Čech nerve projection out of a sufficiently good open cover of $X$ and for $X \stackrel{\simeq}{\leftarrow} C\left(\left\{U_{i}\right\}\right) \stackrel{\text { tw }}{\rightarrow} \mathbf{B}^{3} U(1)$ a cocycle for the twisting circle 3-bundle, a corresponding twisted String-2-bundle is a lift $\hat{g}$ in the diagram of simplicial presheaves

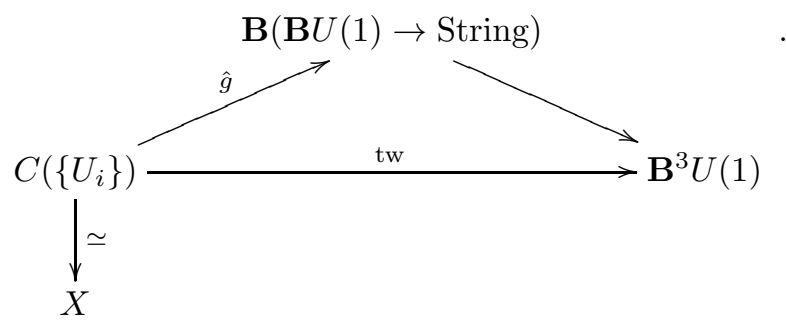

After passing to the differential refinement of this situation, the corresponding Cartan-Ehresmann $L_{\infty}{ }^{-}$ connection is given on $(k+1)$-fold intersections of the cover by compatible diagrams

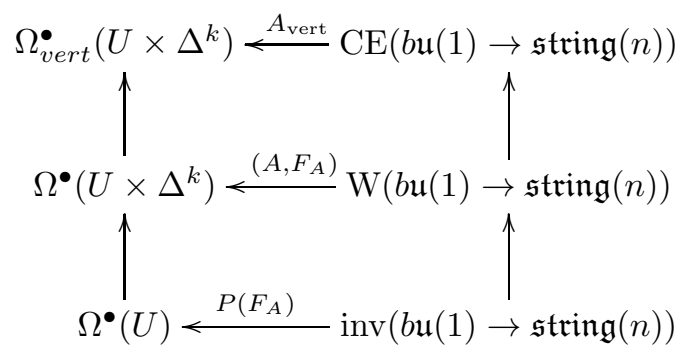

such that the corresponding twist is the prescribed one. If we write $\left(C_{3}\right)$ for the local differential 3 -form data of the prescribed twisting circle 3-bundle, then this means that over $(k+1)$-fold intersections the $L_{\infty}$-algebraic data of a connection on the twisted String-bundle is given by a diagram of dg-algebras of the 
form

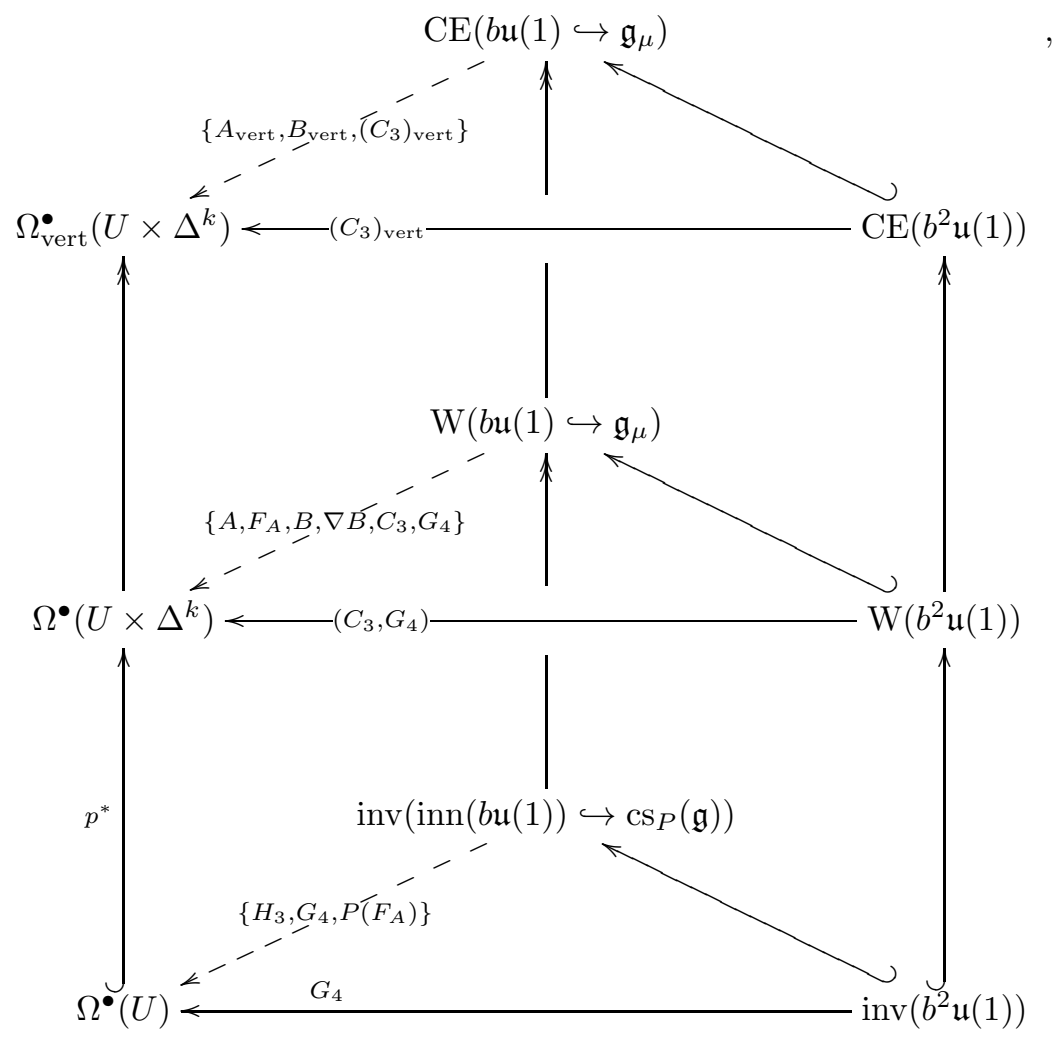

where, for short, we write $\mathfrak{g}_{\mu}$ for $\mathfrak{s t r i n g}$. It may be helpful to think of this as forming sections of a higher associated bundle, as in def. A.10 in the appendix. In terms of this we may read this diagram as indicated 
in the following

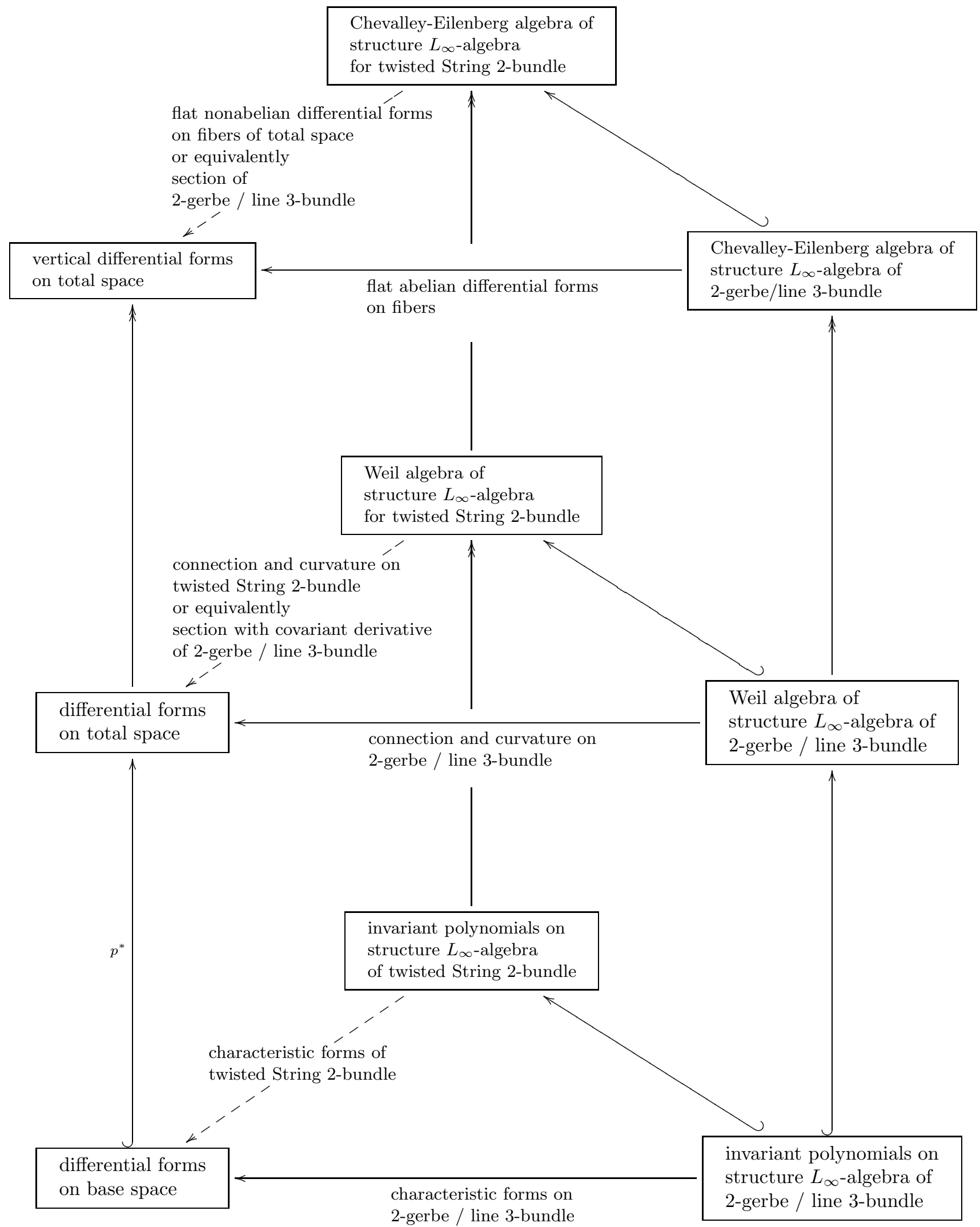


Chasing the generators of the graded-commutative algebras through this diagram and recording the condition imposed by the respect of the morphisms of dg-algebras for differentials, one finds that in components the commutativity of this diagram encodes the following differential form data and the following relations on that.

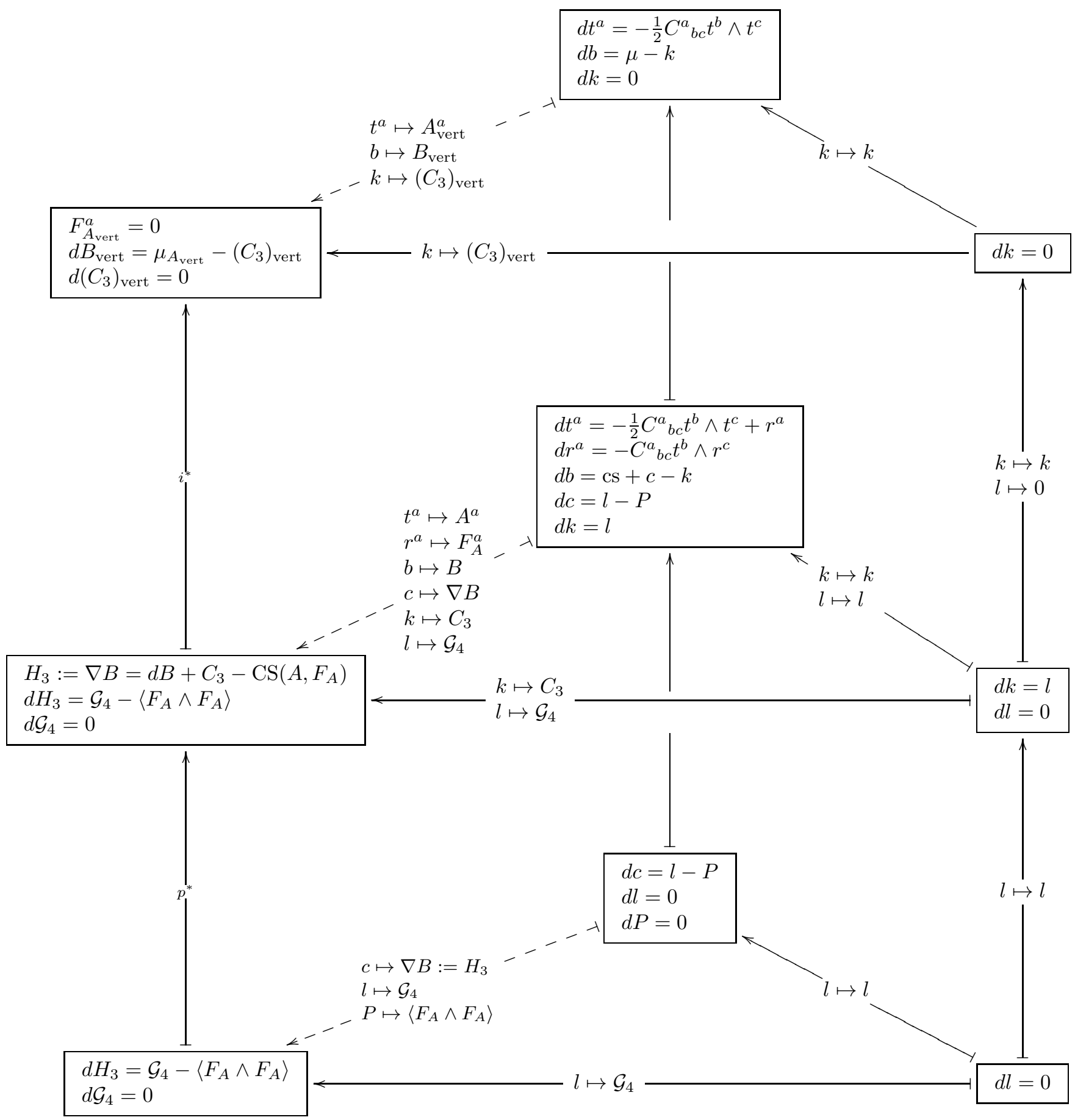


Here, $P \in W(\mathfrak{g})$ denotes the invariant polynomial on $\mathfrak{g}$ in transgression with with the cocycle $\mu \in \operatorname{CE}(\mathfrak{g})$. With $\left\{t^{a}\right\}$ a fixed chosen basis of $\mathfrak{g}^{*}$ in degree 1 and $\left\{r^{a}\right\}$ the corresponding basis in degree 2, we have $P=P_{a b} r^{a} \wedge r^{b}$ and $\mu=\mu_{a b c} t^{a} \wedge t^{b} \wedge t^{c}$ and $\mathrm{cs}=P_{a b} t^{b} \wedge r^{a}+\frac{1}{6} \mu_{a b c} t^{a} \wedge t^{b} \wedge t^{c}$. We have

\begin{tabular}{|l|l|}
\hline curvature & $H_{3}:=d B+C_{3}-\mathrm{CS}\left(A, F_{A}\right)$ \\
\hline Bianchi identity & $d H_{3}=\mathcal{G}_{4}-\left\langle F_{A} \wedge F_{A}\right\rangle$ \\
\hline
\end{tabular}

In [46] this situation was considered from a different perspective for the special case $B=0$ and $\nabla B=0$. There the dashed morphism was obtained as a twisted lift of a $\mathfrak{g}$-connection to a $\mathfrak{g}_{\mu}$-connection and the $b^{2} \mathfrak{u}(1)$-connection appeared as the corresponding obstruction. Here now the perspective is switched: the $b^{2} \mathfrak{u}(1)$-connection is prescribed and the choice of dashed morphisms is a choice of twisted $\mathfrak{g}_{\mu}$-connections with prescribed twist $G_{4}$.

The covariant derivative 3 -form $\nabla B$ of the twisted $\mathfrak{g}_{\mu}$-connection, which we denote by $H_{3}$, measures the difference between the prescribed $b^{2} \mathfrak{u}(1)$-connection and the twist of the chosen twisted $\mathfrak{g}_{\mu}$-connection. The Bianchi identity

$$
d H_{3}=\mathcal{G}_{4}-P\left(F_{A}\right)
$$

which appears in the middle on the left says that this difference has to vanish in cohomology, as one expects. Indeed, this is the structure of the differential forms in the Green-Schwarz mechanism, constituting the differential refinement of the integral cohomology relation (2.10).

3.3. Twisted fivebrane $(n)$ 6-connections. The discussion of twisted differential Fivebrane structures proceeds in direct analogy to the above discussion of twisted differential string structures. One finds that the smooth second fractional Pontryagin class $\frac{1}{6} \mathbf{p}_{2}$ : $\mathbf{B S t r i n g} \rightarrow \mathbf{B}^{7} U(1)$ from the moduli 2-stack of Stringprincipal 2-bundles to the moduli 7-stack of circle 7-bundles is presented by a span of simplicial presheaves of the form

$$
\text { BString } \stackrel{\sim}{\leftarrow} \mathbf{B}\left(\mathbf{B}^{5} U(1) \rightarrow \text { String }\right) \rightarrow \mathbf{B}^{7} U(1)
$$

whose infinitesimal version is a span of $L_{\infty}$-algebras of the form

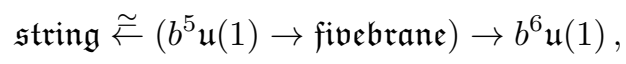

where fivebrane $=\left(\mathfrak{s o}_{\mu_{3}}\right)_{\mu_{7}}$ denotes the Lie 6-algebra from example A.5 and where the middle piece is the mapping cone of the defining extension

$$
b^{5} \mathfrak{u}(1) \rightarrow \text { fibrane } \rightarrow \text { string } .
$$

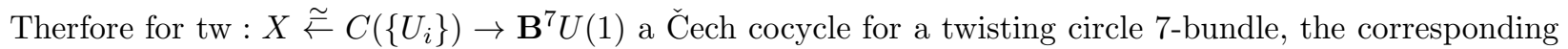
twisted Fivebrane 6-bundles are given by lifts $\hat{g}$ in the disgram of simplicial presheaves

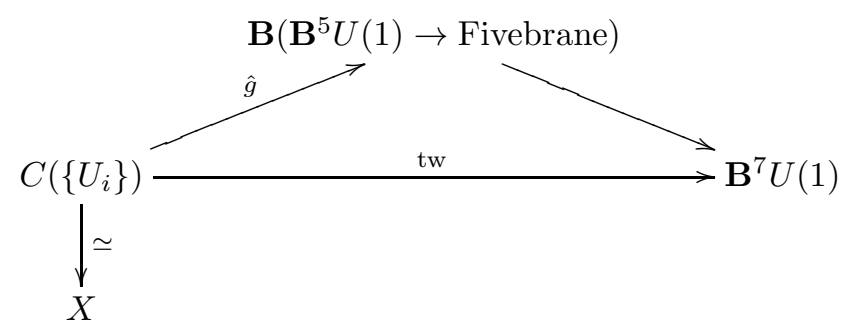

As before, we consider now the analogous diagrams of local $L_{\infty}$-algebra valued forms in order to deduce the local differental form data of twisted differential Fivebrane structures. For transparency of the following diagrams we indicate both the twist of the differential String-structure with local differential forms $\left(C_{3}\right)$ as before, as well as the new twist of the differential Fivebrane structure by local differential forms $\left(C_{7}\right)$. The former has to be taken to vanish, but it is still instructive to display its differential incarnation, for comparison with the previous case. 
Then the local differential cocycle data of a differential refinement of the above twisted Fivebrane structure $\hat{g}$ is given over $k$-fold intersections by a diagram of dg-algebras of the form.

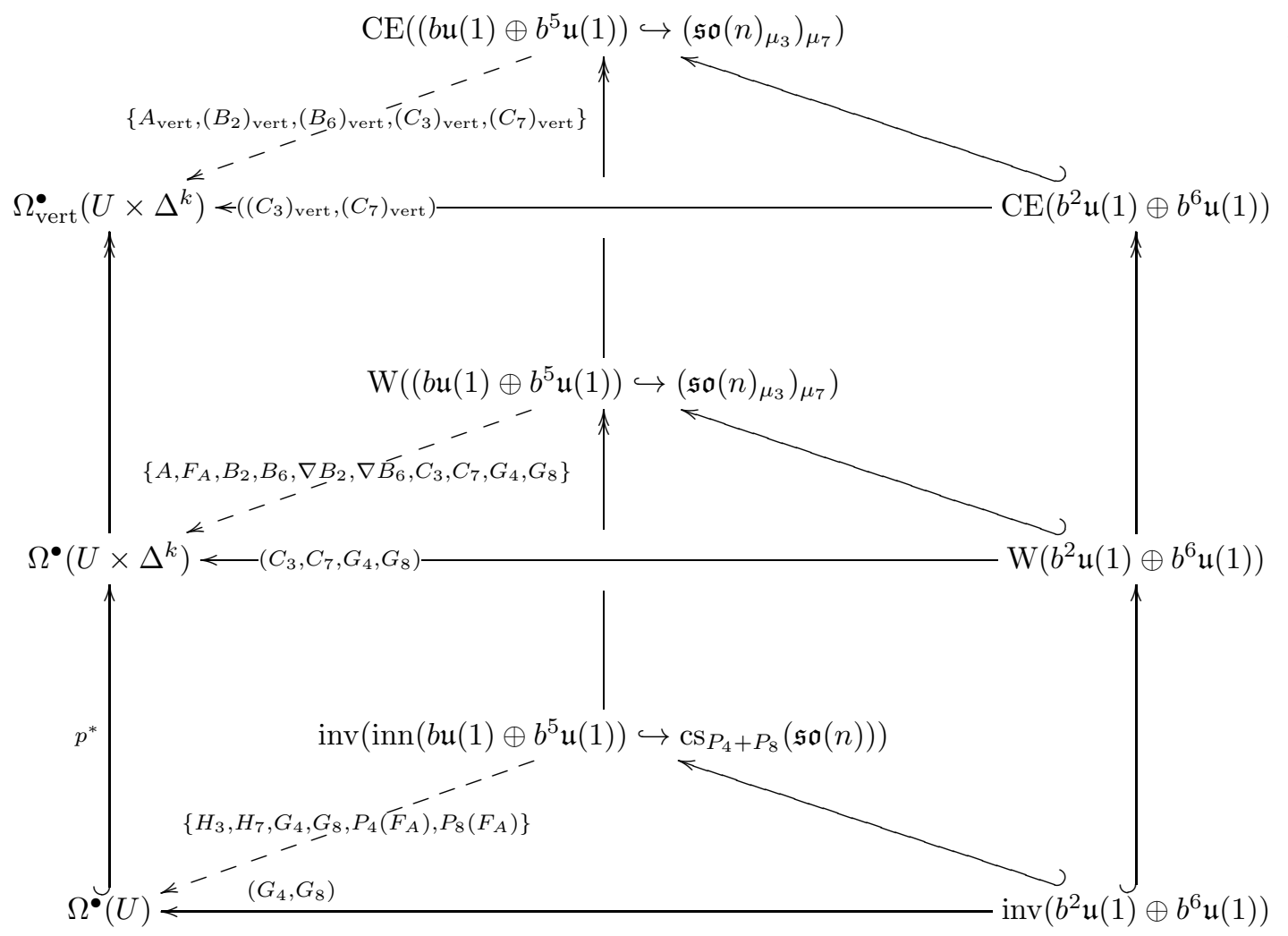


Here is again the meaning in words of the constituents of this diagram:

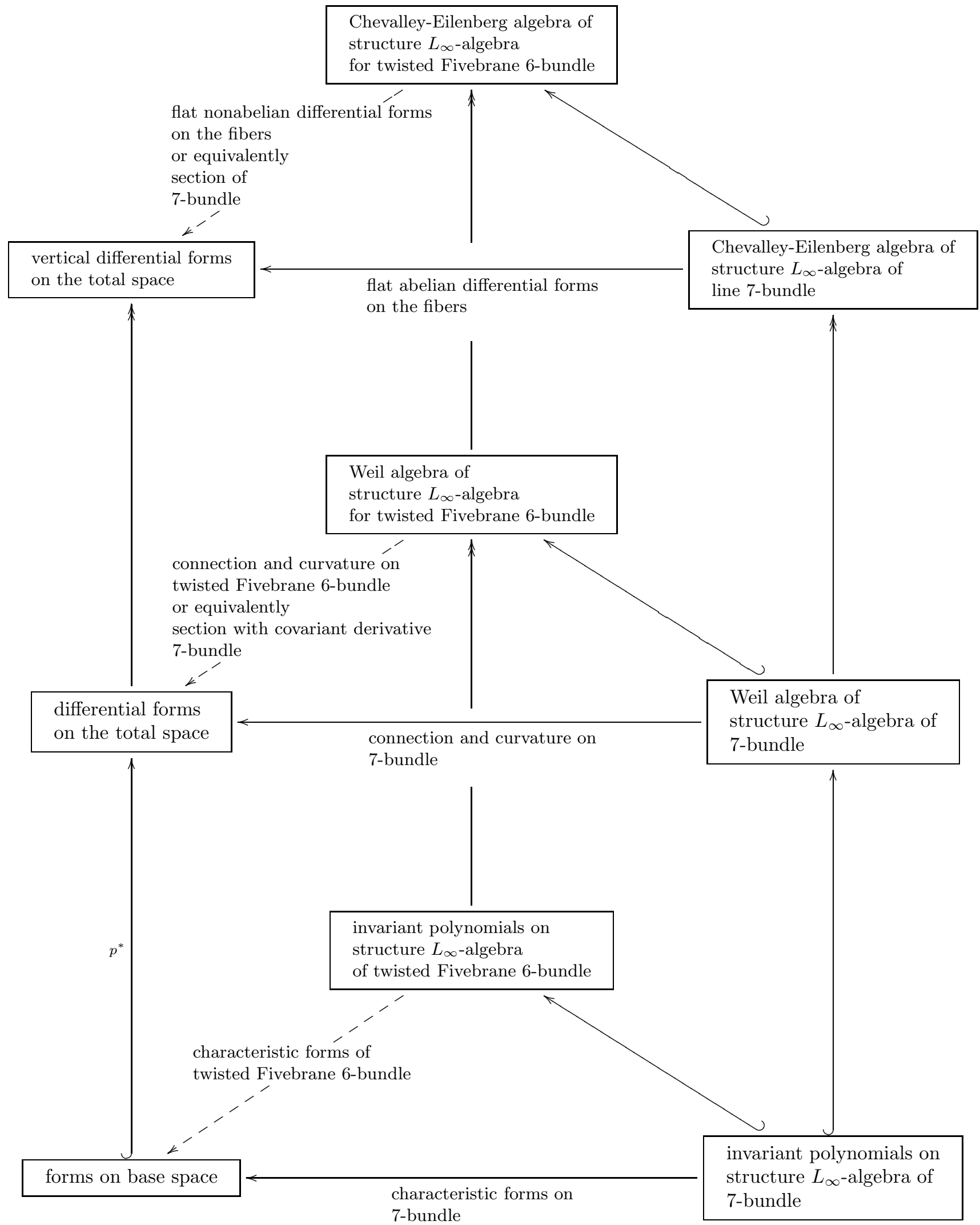


By again chasing elements through the diagram one finds the following data:

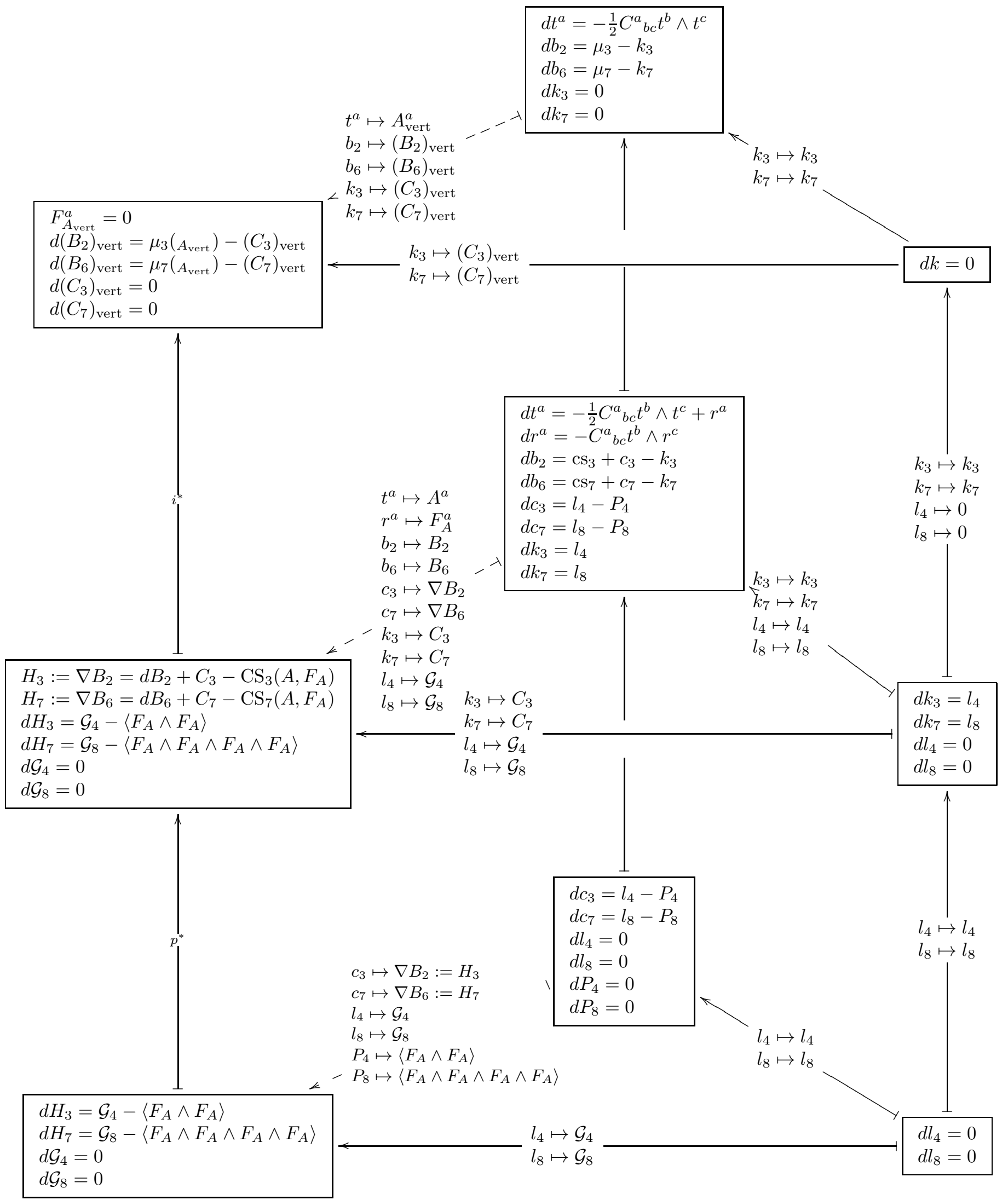


Here, $P_{4}, P_{8} \in W(\mathfrak{g})$ are the invariant polynomials on $\mathfrak{g}$ in transgression with with the cocycles $\mu_{3}, \mu_{7} \in$ $\mathrm{CE}(\mathfrak{g})$. The covariant derivative 7 -form $\nabla B_{6}$ of the twisted $\left(\mathfrak{s o}(n)_{\mu_{3}}\right)_{\mu_{7}}$-connection which we denote by $H_{7}$ measures the difference between the prescribed $b^{6} \mathfrak{u}(1)$-connection and the twist of the chosen twisted $\left(\mathfrak{s o}(n)_{\mu_{3}}\right)_{\mu_{7}}$-connection. The Bianchi identity

$$
d H_{7}=\mathcal{G}_{8}-P_{8}\left(F_{A}\right)
$$

which appears in the middle on the left says that this difference has to vanish in cohomology, being the local incarnation of the differential refinement of the anomaly cancellation condition discussed in section 2.3 .

\section{Appendix A. $L_{\infty}$-ALGEBraic Notions}

We collect here some $L_{\infty}$-algebraic definitions and constructions that are referred to in section 3 Most of the following can be found in more detail in [46], the main point here being the notion of representations of $L_{\infty}$-algebroids and the emphasis of the interpretation of twists as sections, as explained for smooth $\infty$ groupoids in section 3.1. For a more conceptual account of $L_{\infty}$-algebra in the context of smooth $\infty$-groupoids see 49 .

A.1. $L_{\infty}$-Algebras and $L_{\infty}$-Algebroids. In direct generalization of how Lie algebras are infinitesimal approximations to Lie groups, $L_{\infty}$-algebras are infinitesimal approximations to smooth $\infty$-groups. More generally, $L_{\infty}$-algebroids are infinitesimal approximations to smooth $\infty$-groupoids.

Definition A.1. An $L_{\infty}$-algebroid $\mathfrak{a}$ of finite type over a smooth manifold $X$ is a non-positively graded $A:=C^{\infty}(X)$-module degreewise of finite rank, together with a degree +1 derivation

$$
d: \wedge_{A}^{\bullet} \mathfrak{a}^{*} \rightarrow \wedge_{A}^{\bullet} \mathfrak{a}^{*}
$$

linear over the ground field (not necessarily over $A$ ) on the free (over $A$ ) graded-symmetric algebra generated from the $\mathbb{N}$-graded dual $\mathfrak{a}^{*}$ (over $A$ ), such that $d^{2}=0$. The quasi-free (over $A$ ) differential gradedcommutative algebra

$$
\mathrm{CE}(\mathfrak{a}):=\left(\wedge_{A}^{\bullet} \mathfrak{a}^{*}, d\right)
$$

defined this way we call the Chevalley-Eilenberg algebra of the $L_{\infty}$-algebroid $\mathfrak{a}$.

We say the category $L_{\infty}$ Algd of $L_{\infty}$-algebroids is the opposite of the full subcategory of dg-algebras on those of the above form.

It is useful to distinguish the following special cases of this definition.

- For $X=$ pt and $\mathfrak{a}$ concentrated in degree 0 on a vector space $\mathfrak{g}$ we have $\operatorname{CE}(\mathfrak{a})=\left(\wedge \bullet \mathfrak{g}, d_{\mathfrak{g}}\right)$ where $\wedge^{\bullet} \mathfrak{g}$ is the Grassmann algebra on $\mathfrak{g}^{*} d_{\mathfrak{a}}$ is the Chevalley-Eilenberg differential uniquely corresponding to the structure of a Lie algebra on $\mathfrak{g} . L_{\infty}$-algebroids arising this we we write $\mathfrak{a}=b \mathfrak{g}$.

- For $X=\mathrm{pt}$ and $\mathfrak{a}$ concentrated in arbitrary (non-positive) degree the above definition is that of an $L_{\infty}$-algebra structure (of finite type). For $\mathfrak{g}$ any $L_{\infty}$-algebra, we write $\mathfrak{a}=b \mathfrak{g}$ for the $L_{\infty}$-algebroid corresponding to it.

- For $X=$ pt and $d$ at most co-binary (sending generators to wedge products of at most word length 2 in the generators) we have a $d g$-Lie algebra.

- For arbitrary $X$ and $\mathfrak{a}$ concentrated in degree 0 (being finitely generated and projective as a module over $C^{\infty}(X)$ ) this is equivalent to the usual definition of Lie algebroids as vector bundles $E \rightarrow X$ with anchor map [38] $\rho: E \rightarrow T X$ : we have $\mathfrak{g}=\Gamma(E)$ and the anchor is encoded as $\left.d_{\mathfrak{g}}\right|_{C^{\infty}(X)}: f \mapsto \rho(\cdot)(g)$.

- If $\mathfrak{a}$ is concentrated in degrees 0 through $-(n-1)$, then we speak of a Lie $n$-algebroid (Lie $n$-algebra if $X=*$ ).

Example A.2 (line Lie $n$-algebra). For $n \in \mathbb{N}$ let $b^{n} \mathbb{R}$ or equivalently $b^{n} \mathfrak{u}(1)$ be the Lie $n$-algebra defined by the fact that its corresponding Lie $n$-algebroid $b^{n+1} \mathfrak{R}$ has a Chevalley-Eilenberg algebra coming from a single generator in degree $(n+1)$ with vanishing differential. We call this the line Lie n-algebra.

Example A.3 (tangent Lie algebroid). For $X$ a smooth manifold, the de Rham complex $\Omega^{\bullet}(X)$ is the Chevalley-Eilenberg algebra of a Lie 1-algebroid over $X$, called the tangent Lie algebroid $T X, \operatorname{CE}(T X)=$ $\left(\Omega^{\bullet}(X), d_{\mathrm{dR}}\right)$. 
For $\mathfrak{g}$ an $L_{\infty}$-algebra, we say that a closed element $\mu \in \mathrm{CE}(\mathfrak{g})$ of degree $n+1$ is an $(n+1)$-cocycle on $\mathfrak{g}$. Cocycles are equivalently morphisms of $L_{\infty}$-algebras $\mu: \mathfrak{g} \rightarrow b^{n} \mathbb{R}$ or, equivalently, morphisms of $L_{\infty}$ algebroids $\mu: b \mathfrak{g} \rightarrow b^{n+1} \mathbb{R}$.

Example A.4 (higher central extensions). Every cocycle $\mu$ on an $L_{\infty}$-algebra $\mathfrak{g}$ induces a new $L_{\infty}$-algebra, to be denoted $\mathfrak{g}_{\mu}$, which is defined by its CE-algebra being that of $\mathfrak{g}$ with a single generator $b$ in degree $n$ adjoined and the differential extended to this generator by the formula $d_{\mathfrak{g}_{\mu}} b=\mu$. This yields a sequence

$$
b^{n} \mathbb{R} \rightarrow b \mathfrak{g}_{\mu} \rightarrow b \mathfrak{g}
$$

exhibiting the shifted central extension classified by $\mu$.

Example A.5. For $\mathfrak{g}$ a semisimple Lie algebra and $\mu_{3}=\langle-,[-,-]\rangle$ the canonical 3-cocycle, the Lie 2algebra $\mathfrak{g}_{\mu_{3}}$ is called the corresponding String Lie 2-algebra [27, 4]. For $\mathfrak{g}=\mathfrak{s o}$ there is also the canonical 7-cocycle $\mu_{7} \in \mathrm{CE}(\mathfrak{s o})$. This is still a cocycle on $\mathfrak{s o}_{\mu_{3}}$, too, and so there is a Lie 6-algebra $\mathfrak{s o}_{\mu_{3}, \mu_{7}}$, called the fivebrane Lie 6-algebra in [46].

For brevity we state several constructions only for $L_{\infty}$-algebras. The generalization to general $L_{\infty}$ algebroids is immediate. In particular for $\mathfrak{g}$ an $L_{\infty}$-algebra we shall write $\mathrm{CE}(\mathfrak{g})$ as shorthand for $\operatorname{CE}(b \mathfrak{g})$.

Differential form data on a manifold $X$ with values in an $L_{\infty}$-algebra $\mathfrak{g}$ is a graded algebra homomorphism (not necessarily respecting the differentials) from $\mathrm{CE}(\mathfrak{g})$ into the differential forms on $X$ :

$$
\Omega^{\bullet}(X, \mathfrak{g}):=\operatorname{Hom}_{\operatorname{grAlg}}\left(\mathrm{CE}(\mathfrak{g}), \Omega^{\bullet}(X)\right) .
$$

The space of graded algebra homomorphisms is a subspace of the space of linear maps of graded vector spaces from $\mathrm{CE}(\mathfrak{g})$ to $\Omega^{\bullet}(X)$ and, since $\mathrm{CE}(\mathfrak{g})$ is freely generated as a graded algebra and of finite type, this is isomorphic to the space of grading preserving homomorphisms $\operatorname{Hom}_{V e c t[\mathbb{Z}]}\left(\mathfrak{g}^{*}, \Omega^{\bullet}(X)\right)$ from the graded vector space $\mathfrak{g}^{*}$ of dual generators to $\Omega^{\bullet}(X)$. By the usual relation in Vect $[\mathbb{Z}]$ for $\mathfrak{g}$ of finite type, this is isomorphic to the space of elements of total degree 1 in forms tensored with $\mathfrak{g}$ :

$$
\Omega^{\bullet}(X, \mathfrak{g}) \simeq\left(\Omega^{\bullet}(X) \otimes \mathfrak{g}\right)_{0} .
$$

(Recall that $\mathfrak{g}$ is non-positively graded.)

If instead we consider the corresponding homomorphisms of dg-algebras from $\mathrm{CE}(\mathfrak{g})$ into forms, we find that respecting the differentials what deserves to be called flatness

$$
\Omega_{\text {flat }}^{\bullet}(X, \mathfrak{g}):=\operatorname{Hom}_{\operatorname{dgAlg}}\left(\mathrm{CE}(\mathfrak{g}), \Omega^{\bullet}(X)\right) .
$$

The inclusion $\Omega_{\text {flat }}^{\bullet}(X, \mathfrak{g})=\operatorname{Hom}_{\operatorname{dgAlg}}\left(\mathrm{CE}(\mathfrak{g}), \Omega^{\bullet}(X)\right) \longleftrightarrow \Omega^{\bullet}(X) \otimes \mathfrak{g}$ realizes flat $L_{\infty}$-algebra valued forms as elements $A \in \Omega^{\bullet}(X) \otimes \mathfrak{g}$ of forms of total degree 0 with the special property that they satisfy a flatness constraint of the form

$$
d A+\partial A+[A \wedge A]+[A \wedge A \wedge A]+\cdots=0,
$$

where $d$ and $\wedge$ are the operations in $A \in \Omega^{\bullet}(X) \otimes \mathfrak{g}$ and where $[\cdot, \cdot, \cdots]$ are the $n$-ary brackets in the $L_{\infty^{-}}$ algebra and $\partial$ is the differential in the chain complex $\mathfrak{g}$. For $\mathfrak{g}$ a dg-Lie algebra, only the binary bracket is present and $A$ is an ordinary Maurer-Cartan element $D A+[A \wedge A]=0$, where $D=d+\partial$. This equation of course has a long and honorable history in various guises called a Maurer-Cartan equation.

We would like to describe also non-flat $L_{\infty}$-algebra valued forms by homomorphisms of differential graded algebras. This is accomplished by passing from the Chevalley-Eilenberg algebra $\mathrm{CE}(\mathfrak{g})$ to the Weil algebra $\mathrm{W}(\mathfrak{g})$.

Definition A.6. For $\mathfrak{g}$ an $L_{\infty}$-algebra, let $\mathrm{W}(\mathfrak{g})$ be the unique dg-algebra free on the underlying graded vector space $\mathfrak{g}^{*}$ such that the canonical projection morphism $W(\mathfrak{g}) \rightarrow \mathrm{CE}(\mathfrak{g})$ is a dg-homomorphism.

Due to the freeness of $\mathrm{W}(\mathfrak{g})$ we have an isomorphism

$$
\Omega^{\bullet}(X, \mathfrak{g})=\operatorname{Hom}_{\mathrm{grAlg}}\left(\mathrm{CE}(\mathfrak{g}), \Omega^{\bullet}(X)\right) \simeq \operatorname{Hom}_{\operatorname{dgAlg}}\left(\mathrm{W}(\mathfrak{g}), \Omega^{\bullet}(X)\right) .
$$


Definition A.7. The $\infty$-groupoid of $\mathfrak{g}$-valued forms on a smooth manifold $X$ is the Kan complex whose $k$-simplices are $\mathfrak{g}$-valued forms $\Omega_{\mathrm{si}}^{\bullet}\left(X \times \Delta^{k}\right) \leftarrow \mathrm{W}(\mathfrak{g}): A$ on $X \times \Delta^{k}$ with sitting instants (becoming constant perpendicularly towards the faces of the $k$-simplex) and fitting into commutative diagrams of dg-algebras of the form

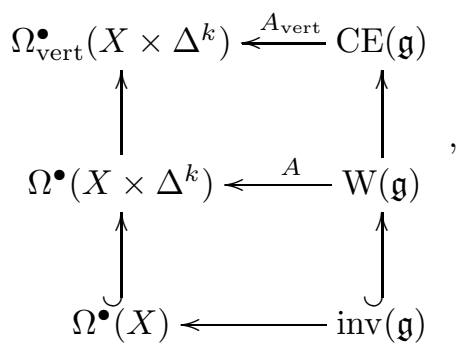

where the vertical morphisms are the canonical ones. For more details on this definition see [21]. The parameterized version of this construction leads to the smooth $\infty$-groupoid $\exp (\mathfrak{g})_{\text {conn }}$ defined in (3.11).

A.2. $L_{\infty}$-algebra representations and section. The $L_{\infty}$-analog of the notion of representations of smooth $\infty$-groupoids as in (3.5) is the following.

Definition A.8 (representations of $L_{\infty}$-algebroids). A representation of an $L_{\infty}$-algebroid $\mathfrak{a}$ over $X$ on a cochain complex $V$ of finite $\operatorname{rank}\left(A:=C^{\infty}(X)\right)$-modules is an $L_{\infty}$-algebroid $V / / \rho_{\rho} \mathfrak{a}$ whose ChevalleyEilenberg algebra $\mathrm{CE}\left(V / /{ }_{\rho} \mathfrak{a}\right)$ is an extension of $\mathrm{CE}(\mathfrak{a})$ by $\wedge_{\mathrm{Sym}_{A} V_{0}^{*}} V^{*}$

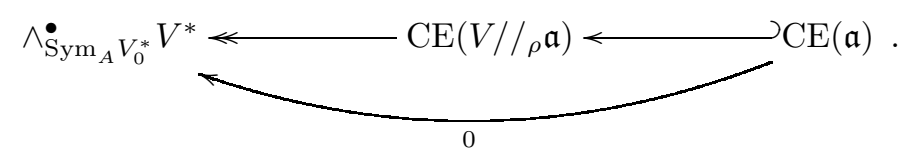

This means that the differential is $\left.d\right|_{\mathfrak{a}^{*}}=d_{\mathfrak{a}},\left.d\right|_{V^{*}}=d_{V^{*}}+d_{\rho}$, where $d_{\rho}$ encodes the action of $\mathfrak{g}$ on $V$.

Remark A.9. In roughly this latter form, the definition appears in [8, where it is called a superconnection. Indeed, in cases where the $L_{\infty}$-algebroid in question is similar to a tangent Lie algebroid of some space, its representations behave like (flat) connections on that space. In the work of [1, for the special case of 1-Lie algebroids, such representations are called representations up to homotopy.

For $\mathfrak{a}=b \mathfrak{g}$ coming from an $L_{\infty}$-algebra, the above notion of representation reproduces the notion of sh-representations of [55] [35].

Example: ordinary adjoint representation. Let $\mathfrak{g}$ be an ordinary Lie algebra with basis $\left\{t_{a}\right\}$ and structure constants $\left\{C^{a}{ }_{b c}\right\}$. Write $\{\underbrace{t^{a}}_{\text {deg=+1 }}\}$ for the corresponding dual basis elements and $\{\underbrace{\chi^{a}}_{\operatorname{deg}=0}\}$ for the corresponding basis elements of $V_{\mathfrak{g}}^{*}$. Then we have $d_{\rho} \chi^{a}=\sigma^{-1}\left(d_{\mathfrak{g}} t^{a}\right)=\sigma^{-1}\left(-\frac{1}{2} C^{a} b c t^{b} \wedge t^{c}\right)=C^{a}{ }_{b c} t^{b} \chi^{c}$.

Definition A.10 (sections and covariant derivatives). Let $\mathfrak{g}$ be an $L_{\infty}$-algebra, and let $V / / \rho \mathfrak{g}$ be a representation of $\mathfrak{g}$, def. A.8. 
Then for $A \in \Omega \bullet\left(X \times \Delta^{k}, \mathfrak{g}\right)$ a $k$-morphism of $\mathfrak{g}$-valued form data on a smooth manifold $X$, def. A.7. we say that a section of the associated $V$-connection is a choice of the dotted arrows in

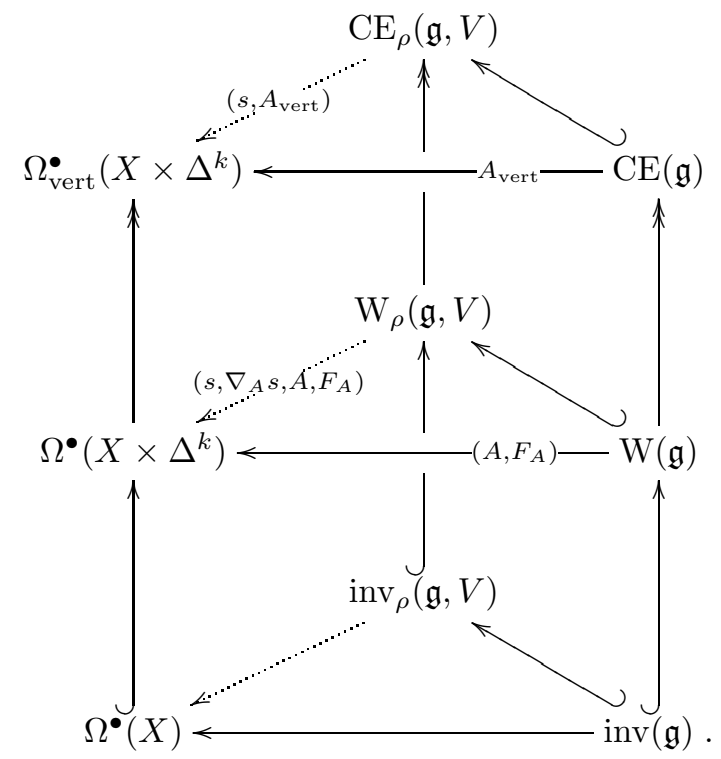

Here we say that $s$ is the section itself whereas $\nabla_{A} s$ is its covariant derivative.

Example A.11. Let $\mathfrak{g}$ be an ordinary Lie algebra with Lie group $G$, let $V$ be a vector space (a chain complex concentrated in degree 0 ) and $\rho$ an ordinary representation of $\mathfrak{g}$ on $V$, let $P$ be a principal $G$-bundle and $\left(A, F_{A}\right)$ an ordinary Cartan-Ehresmann connection on $P$. Then the dotted morphism in

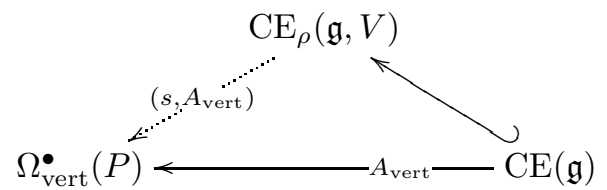

is dual to a $V$-valued function on the total space of the bundle (not on base space!) $s: P \rightarrow V$, which is covariantly constant along the fibers in that the covariant derivative

$$
\nabla_{A} s:=d s+(\rho \circ A) s
$$

vanishes when evaluated on vertical vectors, where $(\rho \circ A) s$ denotes the action of $A$ on the section $s$ using the representation $\rho$. This means that $s$ descends to a section of the associated vector bundle $P \times_{G} V$. The covariant derivative 1 -form $\nabla_{A} s$ of the section $s$ is one component of the extension in the middle part of our diagram

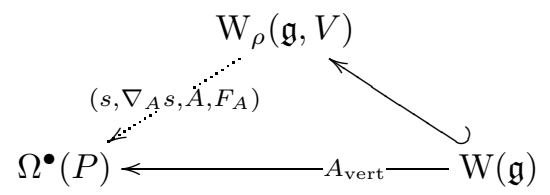

The equation

$$
\nabla_{A} \nabla_{A} s=\left(\rho \circ F_{A}\right) \wedge s
$$

is the Bianchi identity for $\nabla_{A} s$. If $s$ is everywhere non-vanishing, this says that the curvature $F_{A}$ of our bundle is covariantly exact on $P$. In the case that $\mathfrak{g}=\mathfrak{u}(1)$ it follows that $F_{A}$ is an exact 2 -form on $P$ and the choice of the non-vanishing section amounts to a trivialization of the bundle. 
In sections 3.2 and 3.3 we see twisted differential String-structures and twisted differential Fivebrane structures as parameterized examples of this notion of $L_{\infty}$-sections.

\section{Acknowledgements}

H. S. and U. S. would like to thank the Hausdorff Institute for Mathematics in Bonn for hospitality and the organizers of the "Geometry and Physics" Trimester Program at HIM for the inspiring atmosphere during the initial stages of this project. H.S. thanks Matthew Ando for useful discussions. U. S. also thanks the Max-Planck institute for Mathematics in Bonn for hospitality later during this work; and the crew of the $n \mathrm{Lab}$, where some of the material presented here was first exposed. This research is supported in parts by the FQXi mini-grant "QFT and Nonabelian Differential Cohomology" and NSF Grant PHY-1102218. H. S. thanks the Department of Mathematics at Hamburg University for hospitality during the writing of this paper. J. S. would like to thank the Department of Mathematics of the University of Pennsylvania for support of the Deformation Theory Seminar enabling the three authors to have at least one meeting in person. The authors are indebted to the referee for many useful remarks and suggestions that led to major improvements of the paper.

\section{REFERENCES}

[1] C. Abad and M. Crainic, Representations up to homotopy of Lie algebroids, arXiv:0901.0319] [math.DG].

[2] P. Aschieri and B. Jurčo, Gerbes, M5-brane anomalies and $E_{8}$ gauge theory, J. High Energy Phys. 0410 (2004) 068, arXiv:hep-th/0409200.

[3] N. A. Baas, M. Bökstedt, and T. A. Kro, Two-categorical bundles and their classifying spaces, arXiv:math/0612549 [math.AT].

[4] J. Baez, A. Crans, U. Schreiber, and D. Stevenson, From loop groups to 2-groups, Homology, Homotopy Appl. 9 (2007), no. 2, 101- 135, arXiv:math/0504123 [math.QA].

[5] J. Baez, and U. Schreiber, Higher gauge theory, Categories in Algebra, Geometry and Mathematical Physics, 7-30, Contemp. Math., 431, Amer. Math. Soc., Providence, RI, 2007, arXiv:math/0511710v2] [math.DG].

[6] J. Baez and D. Stevenson, The classifying space of a topological 2-group, arXiv:0801.3843] [math.AT].

[7] K. Behrend, P. Xu, Differentiable Stacks and Gerbes, J. Symplectic Geom. 9 (2011), 285-341, arXiv:math/0605694].

[8] J. Block, Duality and equivalence of module categories in noncommutative geometry I, arXiv:math/0509284] [math.QA].

[9] R. Bott, The space of loops on a Lie group, Michigan Math. J., 5:3561, (1958)

[10] P. Bouwknegt, A. L. Carey, V. Mathai, M. K. Murray, and D. Stevenson, Twisted K-theory and K-theory of bundle gerbes, Commun. Math. Phys. 228 (2002) 17-49, arXiv:hep-th/0106194 2].

[11] P. Bouwknegt and V. Mathai, D-branes, B-fields and twisted K-theory, J. High Energy Phys. 03 (2000) 007, arXiv:hep-th/0002023.

[12] J. Brodzki, V. Mathai, J. Rosenberg, and R. J. Szabo, D-branes, RR-fields and duality on noncommutative manifolds, Commun. Math. Phys. 277, no.3 (2008) 643-706, arXiv:hep-th/0607020 33].

[13] U. Bunke, String structures and trivialisations of a Pfaffian line bundle, arXiv:0909.0846

[14] A. H. Chamseddine, Interacting supergravity in ten-dimensions: the role of the six-index gauge field, Phys. Rev. D24 (1981) 3065.

[15] A. H. Chamseddine, A family of dual $N=2$ supergravity actions in ten-dimensions, Phys. Lett. B367 (1996) 134-139, arXiv:hep-th/9510100.

[16] E. Diaconescu, D. S. Freed and G. Moore, The M-theory 3-form and E8 gauge theory, Elliptic cohomology, 44-88, London Math. Soc. Lecture Note Ser., 342, Cambridge Univ. Press, Cambridge, 2007, arXiv:hep-th/0312069.

[17] E. Diaconescu, G. Moore and E. Witten, $E_{8}$ gauge theory, and a derivation of K-theory from M-theory, Adv. Theor. Math. Phys. 6 (2003) 1031, arXiv:hep-th/0005090.

[18] J. A. Dixon, M. J. Duff, and J. C. Plefka, Putting string/fivebrane duality to the test, Phys. Rev. Lett. 69 (1992) 3009-3012, arXiv:hep-th/9208055 1 1].

[19] M. J. Duff, J. T. Liu, and R. Minasian, Eleven-dimensional origin of string-string duality: A one loop test, Nucl. Phys. B452 (1995) 261-282, arXiv:hep-th/9506126.

[20] J. Evslin and H. Sati, Can D-branes wrap nonrepresentable cycles?, J. High Energy Phys. 0610 (2006), 050, arXiv:hep-th/0607045.

[21] D. Fiorenza, U. Schreiber, J. Stasheff, Čech cocycles for differential characteristic classes - an $\infty$-Lie theoretic construction, arXiv: 1011.4735

[22] D. S. Freed, Dirac charge quantization and generalized differential cohomology, Surv. Differ. Geom., VII, 129-194, Int. Press, Somerville, MA, 2000, arXiv:hep-th/0011220.

[23] D. Freed, J. A. Harvey, R. Minasian, and G. Moore, Gravitational anomaly cancellation for M-theory fivebranes, Adv. Theor. Math. Phys. 2 (1998) 601-618, arXiv:hep-th/9803205. 
[24] D. S. Freed and E. Witten, Anomalies in string theory with D-Branes, Asian J. Math. 3 (1999) 819, arXiv:hep-th/9907189.

[25] S.J. Gates, Jr., and H. Nishino, New $D=10, N=1$ supergravity coupled to Yang-Mills supermultiplet and anomaly cancellations, Phys. Lett. B157 (1985) 157.

[26] M. B. Green and J. H. Schwarz, Anomaly cancellation in supersymmetric D 10 gauge theory and superstring theory, Phys. Lett. B149 (1984) 117.

[27] A. Henriques, Integrating $L_{\infty}$-algebras, Compos. Math. 144 (2008), no. 4, 1017-1045, arXiv:math/0603563 [math.AT].

[28] M. J. Hopkins and I. M. Singer, Quadratic functions in geometry, topology, and M-theory, J. Diff. Geom. 70 (2005) 329-452, arXiv:math.AT/0211216.

[29] P. Horava and E. Witten, Eleven-dimensional supergravity on a manifold with boundary, Nucl. Phys. B475 (1996) 94-114, arXiv:hep-th/9603142.

[30] C.J. Isham and C.N. Pope, Nowhere vanishing spinors and topological obstructions to the equivalence of the NSR and GS superstrings, Class. Quant. Grav. 5 (1988) 257.

[31] C.J. Isham, C.N. Pope, and N.P. Warner, Nowhere vanishing spinors and triality rotations in eight manifolds, Class. Quant. Grav. 5 (1988) 1297.

[32] A. Kapustin, D-branes in a topologically nontrivial B-field, Adv. Theor. Math. Phys. 4 (2000) 127, arXiv:hep-th/9909089.

[33] T. P. Killingback, World-sheet anomalies and loop geometry, Nucl. Phys. B288 (1987) 578.

[34] L. Kramer and S. Stolz, A diffeomorphism classification of manifolds which are like projective planes, J. Differential Geom. 77 (2007), no. 2, 177-188, arXiv:math/0505621 v3] [math.GT].

[35] T. Lada and M. Markl, Strongly homotopy Lie algebras, Comm. Algebra 23 (1995), no. 6, 2147-2161.

[36] K. Lechner and M. Tonin, World volume and target space anomalies in the $D=10$ superfivebrane sigma model, Nucl. Phys. B475 (1996) 545-561, arXiv:hep-th/9603094.

[37] J. Lurie, Higher topos theory, Annals of mathematics studies, no. 170, Princeton University Press, Princeton, NJ, 2009, arXiv:math/0608040 [math.CT].

[38] K. C. H. Mackenzie, General theory of Lie groupoids and Lie algebroids, Cambridge University Press, Cambridge, 2005.

[39] T. Nikolaus, C. Sachse, C. Wockel, A smooth model for the string 2-group, arXiv:1104.4288

[40] H. Nishino and S. J. Gates, Jr., Dual versions of higher dimensional supergravities and anomaly cancellations in lower dimensions, Nucl. Phys. B268 (1986) 532-542.

[41] C. Redden String structures and canonical 3-forms, arXiv:0912.2086] [math.DG].

[42] A. Salam and E. Sezgin, Anomaly freedom in chiral supergravities, Phys. Scripta 32 (1985) 283.

[43] H. Sati, Geometric and topological structures related to M-branes, Proc. Symp. Pure Math. 81 (2010), 181-236, arXiv:1001.5020 [math.DG].

[44] H. Sati, Geometric and topological structures related to M-branes II: Twisted String and String ${ }^{c}$ structures, J. Australian Math. Soc. 90 (2011), 93-108, arXiv:1007.5429] [hep-th].

[45] H. Sati, Twisted topological structures related to M-branes, Int. J. Geom. Meth. Mod. Phys. 8 (2011), 1097-1116, arXiv: 1008.1755 [hep-th].

[46] H. Sati, U. Schreiber and J. Stasheff, $L_{\infty}$-connections and applications to String- and Chern-Simons n-transport, in Recent Developments in QFT, eds. B. Fauser et al., Birkhäuser, Basel (2008), arXiv:0801.3480] [math.DG].

[47] H. Sati, U. Schreiber, and J. Stasheff, Fivebrane structures, Rev. Math. Phys. 21 (2009) 1-44, [arXiv:math/0805.0564] [math.AT].

[48] C. Schommer-Pries A finite-dimensional model for the String 2-group, arXiv:0911.2483] [math.AT].

[49] U. Schreiber. Differential cohomology in a cohesive topos, Habilitation, Hamburg (2011) http://ncatlab.org/schreiber/show/differential+cohomology+in+a+cohesive+topos

[50] U. Schreiber, On $\infty$-Lie theory, http://www.math.uni-hamburg.de/home/schreiber/action.pdf].

[51] U. Schreiber and K. Waldorf, Parallel transport and functors, arXiv:0705.0452] [math.DG].

[52] U. Schreiber and K. Waldorf, Smooth functors vs. differential forms, arXiv:0802.0663] [math.DG].

[53] U. Schreiber and K. Waldorf, Connections on nonabelian gerbes and their holonomy, arXiv:0808.1923. [math.DG].

[54] W. M. Singer, Connective fiberings over BU and U, Topology 7 (1968) 271-303.

[55] J. Stasheff, Constrained Poisson algebras and strong homotopy representations, Bull. Amer. Math. Soc. (N.S.) 19 (1988), no. 1, 287-290.

[56] S. Stolz and P. Teichner, What is an elliptic object?, in Topology, geometry and quantum field theory, 247-343, Cambridge Univ. Press, Cambridge, 2004.

[57] R. Stong, Determination of $H^{*}\left(\mathrm{BO}(k, \cdots, \infty), Z_{2}\right)$ and $H^{*}\left(\mathrm{BU}(k, \cdots, \infty), Z_{2}\right)$, Trans. Amer. Math. Soc. $107(1963)$ $526-544$.

[58] B. Toën, Notes on non-abelian cohomology, Lecture in MSRI, January 2002, http://www.math.univ-toulouse.fr/ toen/msri2002.pdf.

[59] C. Vafa and E. Witten, A one-loop test of string duality, Nucl. Phys. B447 (1995) 261-270, arXiv:hep-th/9505053.

[60] K. Waldorf, String connections and Chern-Simons theory, arXiv:0906.0117] [math.DG].

[61] B.-L. Wang, Geometric cycles, index theory and twisted K-homology, J. Noncommut. Geom. 2 (2008), no. 4, 497-552, arXiv:0710.1625 1 ] [math.KT].

[62] F. Witt, Special metric structures and closed forms, DPhil Thesis, University of Oxford, 2004. 
[63] E. Witten, On flux quantization in M-theory and the effective action, J. Geom. Phys. 22 (1997) 1-13, arXiv:hep-th/9609122.

[64] E. Witten, Five-brane effective action in M-theory, J. Geom. Phys. 22 (1997) 103-133, arXiv:hep-th/9610234.

[65] E. Witten, D-Branes and K-Theory, J. High Energy Phys. 12 (1998) 019, arXiv:hep-th/9810188.

[66] E. Witten, Duality relations among topological effects in string theory, J. High Energy Phys. 0005 (2000) 031, arXiv:hep-th/9912086.

Department of Mathematics, Yale University, New Haven, CT 06511

Current address: Department of Mathematics, University of Maryland, College Park, MD 20742

E-mail address: hsati@math.umd.edu

Fachbereich Mathematik, Universität Hamburg, Bundesstrasse 55, D-20146 Hamburg, Germany

Current address: Department of Mathematics, Utrecht University, 3508 TA Utrecht, The Netherlands

E-mail address: schreiber@math.uni-hamburg.de

Department of Mathematics, University of Pennsylvania, David Rittenhouse Lab, Philadelphia, PA 19104-6395

E-mail address: jds@math.upenn.edu 